\title{
LA VOCE DEI POETI ALLA CORTE ARAGONESE. LA FESTA E IL TEATRO
}

\section{Francesca Bortolettit}

\begin{abstract}
Riassunto: Nella Napoli dei Re d'Aragona (1442-1504) le presenze della poesia orale nei luoghi dell'intrattenimento umanistico e del teatro aragonese giocano un ruolo determinante nel cerimoniale cortigiano e nelle strategie di politica culturale del Regno Aragonese. Il presente studio mira a rintracciare esempi di scritture, storie e materiali legati a una recitazione estemporanea replicabile in diverse occasioni di cerimoniali di corte o degli episodi politici. Una recitazione che non può essere compresa se non messa in relazione con una visione unitaria della festa di corte che qui si propone di investigare attraverso le presenze della voce del 'nuovo' poeta umanista — diplomatico e oratore, storico e cerimoniere, poeta e performer - e delle sue pratiche dicitorie e recitative nella società di corte.
\end{abstract}

La vicenda politica degli Aragona a Napoli (1442-1504) coincide storicamente con una fase decisiva di formazione della letteratura volgare e dell' invenzione' del teatro nello scenario quattrocentesco italiano. Napoli, tra la seconda metà del Quattrocento e l'inizio del nuovo secolo mostra, rispetto alla sperimentazione della cerchia medicea o al modello ferrarese e romano, una situazione di apparente ritardo, non presentando da un lato un'identità letteraria uniforme e dall'altro una cultura teatrale indirizzata alla attualizzazione delle forme tradizionali classiche, mostrandosi caratterizzata invece da una varietas e una contaminazione di lingue, stili e tradizioni di culture straniere, peninsulari e autoctone. Sebbene l'umanesimo napoletano sia originariamente frutto di una cultura d'importazione, come notava Galasso, piuttosto che di una maturazione lenta e spontanea, con il nuovo governo

\footnotetext{
* La ricerca che ha portato a questa pubblicazione si è svolta all'interno del progetto Italian Voices. Oral Culture, Manuscript and Print in Early Modern Italy, diretto dal prof. Brian Richardson, e ha ricevuto i finanziamenti dall'European Research Council sotto il Settimo Programma Quadro dell'Unione Europea (FP7/2007-2013) / ERC Grant Agreement n. 269460. Un ringraziamento sincero va a Brian Richardson e Paola Ventrone per i loro preziosi commenti e suggerimenti.
} 
aragonese e il cambio degli equilibri interni e peninsulari, la città diviene, sotto la guida di Alfonso il Magnanimo (1396-1458) e dei suoi successori, un importante centro di cultura, fortemente plurilingue, ma contraddistinto da una classe di intellettuali a stretto contatto con gli uomini di potere, le cui opere riflettevano la società politica a cui appartenevano. ${ }^{1}$ In questi anni le tradizioni di spettacolo di matrice ancora cortese e cavalleresca del periodo medievale, s'incontrano con le misurate innovazioni avanzate da questa fervida cerchia di intellettuali e poeti latini e volgari gravitante intorno all'Accademia Pontaniana e coinvolta nella politica festiva dei re e dei duchi del regno. ${ }^{2}$

La presenza di questi poeti è progressivamente registrata durante le tradizionali occasioni festive, per patti matrimoniali, nozze, battesimi o incoronazioni - contraddistinte per lo più da trionfi, giostre, tornei e cacce — ma anche nei luoghi dell'intrattenimento privato e nelle consuetudini associative e conviviali a corte e in accademia. All'interno del tradizionale cerimoniale il loro operare come diplomatici, cerimonieri e insieme poeti e intrattenitori dello spettacolo privato

${ }^{1}$ Galasso, "Introduzione" alla trad. it di Bently (Politica e cultura nella Napoli rinascimentale), 7-11 — da cui qui e di seguito si cita. Nell'ambito degli studi rinascimentali il Regno di Napoli non ha goduto di grande attenzione specie se paragonato alle altre corti del centronord. Nel prezioso lavoro di Bentley, lo studioso mira a riconsiderare la vicenda aragonese dagli anni della conquista (1435-1442) fino alla discesa di Carlo VIII e all'imposizione del regno diretto della Spagna (1504) in una prospettiva non solo locale ma anche nazionale, osservando il ruolo determinante giocato dal Regno nella politica peninsulare. Su Alfonso I il Magnanimo si veda Pontieri; Ryder, The kingdom of Naples under Alfonso the Magnanimo. Si veda inoltre il ritratto che di lui ha lasciato Antonio Beccadelli, il Panormita nel De dictis et factis Alphonsi regis Aragonum (1455) testo latino dell'opera consultabile in internet: http://www.bibliotecaitaliana. it, dell'Università degli studi di Roma.

${ }^{2}$ Oltre al Magnanimo e a Ferrante, figure di primo piano furono il duca Alfonso di Calabria e la raffinata consorte Ippolita Sforza, donna "in eloquio facunda et eloquente" — come la ricorda Sabadino degli Arienti - giunta nel Regno nel 1465 e celebrata da suntuose nozze. Ippolita Sforza, figlia di Francesco Sforza e Bianca Maria Visconti, assunse presto un ruolo significativo nella politica culturale aragonese e fu un importante tramite nelle relazioni con la corte paterna e le corti del nord, ma anche con il circolo dei poeti medicei. Attorno a lei gravitarono alcuni dei poeti volgari più avvertiti che guardavano con interesse alla produzione del nord. Nella Vita Sabadino la ricorda tra le altre cose per le sue grandi virtù oratorie su argomenti vari, ma soprattutto sulle 'questioni' di politica 'interna' e 'estera.' Sabbadino Degli Arienti, 339 e 345. Si veda anche l'edizione delle Lettere inedite di Joviano Pontano in nome de' reali di Napoli, che contiene alcune lettere di Ippolita; e ancora i seguenti studi: Welch; Bryce e ivi bibliografia interna; Soranzo 2009. 
e pubblico di corte affinò una produzione di poesia recitativa d'occasione di tipo amoroso, gnomico e morale che, inserita all'interno del progetto unitario della festa di corte e della sua dimensione politica, caricava l'immagine del poeta-performer di nuovi valori elaborati dalla cultura umanistica. Fissava inoltre i canoni di un repertorio drammaturgico complesso e vario, in cui la componente letteraria e musicale, che traeva alimento dalla tradizione petrarchesca e insieme dalle forme autoctone di poesia solo apparentemente popolareggiante, si univa a quella coreografica e visiva.

A Napoli, le presenze della poesia orale nei luoghi dell'intrattenimento elitario e le pratiche di teatro aragonese giocano infatti un ruolo determinante. Si collocano all'interno dei sistemi di comunicazione del tradizionale cerimoniale cortigiano e delle strategie di politica culturale impegnate nella creazione della magnificentia del Regno Aragonese - celebrata anche dalla trattatistica, dal Panormita, dal Facio, dal Pontano e dal De Maio.

Dopo gli studi ancora preziosi — sia pur ormai lontani — di Torraca e Croce sulle molteplici forme della festività nella Napoli aragonese, ${ }^{3}$ nuovi contributi hanno sensibilmente arricchito il quadro critico e documentario sulla dimensione festiva e le pratiche recitative coeve,${ }^{4}$ che tuttavia si presenta ancora limitato specie se comparato allo stato degli studi sulle corti di area pavana, di Venezia, di Firenze, Urbino o Roma tra Quattro e Cinquecento. ${ }^{5}$ Ricerche puntuali, inoltre, sulla produzione testuale di farse, ${ }^{6}$ inframesse e gliommeri, ${ }^{7}$ e ancora sull'egloga pastorale, ${ }^{8}$ hanno messo in evidenza un repertorio di testi eterogeneo e una progressiva em-

\footnotetext{
${ }^{3}$ Torraca 1884; Croce 1891.

${ }^{4}$ Fondamentali sono gli studi di Marzia Pieri (Pieri 1985) e il recente volume di Cristiana Anna Addesso (Addesso 2012).

${ }^{5}$ Sul valore concettuale riconosciuto alla festa si rimanda, per l'area italiana, ai contributi di Cruciani 1987 e Cruciani 1983; Zorzi; Guarino 1988; Ventrone 1992.

${ }^{6}$ Per i testi delle farse pervenute si veda l'edizione Sannazaro, Opere volgari. Cfr. anche i saggi critici di: Bersani 1982; Bersani 1982 (“Alla ricerca...”); Bersani 1983; De Blasi 1993. Più recenti i contributi di Nocilli; e Bianchi.

${ }^{7}$ Torraca 1925; Croce 1916; Parenti 1978; De Blasi 1999; De Blasi 2009.

${ }^{8}$ Pieri 1985; Pieri 1993; si veda anche Laura Riccò sulla fortuna di “microstrutture napoletane," in Riccò, 27-42. Desidero ringraziare l'autrice per il dono di una lettura in anteprima del suo volume. Per una ricognizione sull'egloga volgare quattrocentesca e le sue relazioni con le culture della performance nella società umanistica e rinascimentale cfr. Bortoletti 2008; si veda anche il prezioso e ricco studio in ambito musicologico di Gerbino 2009.
} 
ersione di un volgare illustre napoletano che merita di essere ripreso e osservato in relazione alle dinamiche dello spettacolo di corte e dell'intrattenimento elitario.

Il presente studio mira a rintracciare esempi di scritture, storie e materiali legati a una recitazione estemporanea di moduli tematici e testuali collaudati e replicabili in diverse occasioni di cerimoniali di corte o degli episodi politici. Una recitazione che non può essere compresa se non messa in relazione con una visione unitaria della festa di corte, che qui si propone di investigare attraverso le presenze nei luoghi di potere della voce del 'nuovo' poeta umanista - diplomatico e oratore, storico e cerimoniere, poeta e performer —, e delle sue pratiche dicitorie e recitative nella società di corte.

\section{Poesia recitativa e festa}

Nella Napoli degli Aragona le occasioni cerimoniali e festive si intensificano sensibilmente elaborando una progettualità spettacolare. Tale progettualità, sebbene si manifesti in una forma ancora poco organica e coerente, mostra in realtà, a partire dalla fine degli anni Settanta, una progressiva elaborazione da parte di poeti illustri e rimatori di un repertorio poetico per la scena e di un originale teatro parlato e cantato. Si tratta di una produzione poetica e recitativa che, come si accennava in apertura, è stata spesso declassata anche dalla critica più avvertita come arretrata rispetto ai linguaggi teatrali delle altre corti peninsulari, ma che tuttavia sarà in non pochi casi modello per il nuovo teatro cortigiano.

A Napoli, nelle residenze reali — fra tutte: Castelnuovo, Castel Capuano e a partire dagli anni Novanta la villa di Poggioreale costruita con il progetto di Giuliano da Maiano per la duchessa Ippolita - erano predisposte ampie sale con preziosi arazzi per le celebrazioni solenni, ma furono anche adibite apposite stanze, "cambre della musica," provviste di organi e altri strumenti a diletto quotidiano della corte. ${ }^{\text {? }}$

Giovanni Pontano ricorda una socialità cortigiana che a Napoli sin dai tempi di Alfonso il Magnanimo creava situazioni di intrattenimento e piacere nell'ascolto delle recite (o letture) dei dotti poeti e fra tutti del Panormita.

Rex Alfonsus statim post prandium vel Antonium Panhormitam, vel e doctis aliquem audiebat, ut qui dignum iudicaret animum quoque cibo suo post pastum corporis reficiendum esse.

\footnotetext{
${ }^{9}$ Cfr. Atlas, 101. Cfr. anche Anglés.
} 
Trad. Il re Alfonso subito dopo pranzo ascoltava Antonio Panormita o qualcuno degli altri dotti, perché riteneva giusto che si dovesse rifocillare l'animo con il suo proprio cibo, dopo aver nutrito il corpo. ${ }^{10}$

Questa nota del Pontano trova conferma anche nel racconto di Adamo di Montaldo che, in un'orazione in lode di Alfonso, riferisce delle abitudini del re di ritirarsi nella Biblioteca per dilettarsi nell'ascoltare le dispute poetiche e letterarie dei 'suoi' dotti umanisti. Insieme alla corte (e alla sua funzione di patronato), la Biblioteca reale giocò infatti un ruolo determinante nell'affermazione dell'umanesimo aragonese. ${ }^{11} \mathrm{Ma}$ l'amore per le dispute letterarie sugli antichi accompagnava, nella 'leggenda magnanima' elaborata con scrupolo dai suoi umanisti, anche le spedizioni militari. Lo racconta il Panormita nel suo De dictis et factis Alphonsi, ricordando, ad esempio fra le mille storie, la disputa sull'eroe portoghese Viriato, che egli intrattenne con Alfonso sul campo militare di Caiazzo (1441), prima della battaglia, in uno dei padiglioni sontuosamente arredati, e che il Magnanimo non volle interrompere finché questa non si fosse conclusa: "Ove si tratti di questioni di letteratura o di storia antica — disse Alfonso — non c'è spazio per uomini d'armi ma per uomini di toga" ("locum [...] cum de literis, aut cognitione antiquitatis ageretur non purpuratorum, sed togatorum esse"). ${ }^{12}$

E ancora il Pontano rammenta l' "immenso splendore” dei banchetti privati e delle feste pubbliche in cui si alternavano meravigliosi spettacoli e competizioni poetiche o performances recitative che necessitavano dell'impiego di artisti dello spettacolo:

Institutum etiam fuit regnum Neapolitanorum annis singulus, statis quibusdam diebus, papere nobilitati epulum ad aedem Mariae

\footnotetext{
${ }^{10}$ Pontano, “De Coviventia,” II, p. 256. Ma si veda sempre in Pontano 2003 (il De Principe) pp. $14,26,28,34$.

${ }^{11}$ La Biblioteca, allestita prima a Castel Capuano poi, dopo il restauro, a Castelnuovo, contava di un'ampia raccolta di volumi e vantava un cospicuo numero di copisti e miniatori quotidianamente impiegati nella sua valorizzazione. Essa fu, inoltre, sede di letture e dispute letterarie e luogo di formazione di un'eletta gioventù napoletana a sostituzione, in certi tempi, dello Studio. Vespasiano da Bisticci ricorda nelle Vite che Alfonso spese più di ventimila ducati per lo Studio. Tuttavia, solo durante il regno di Ferrante, questo ebbe un ruolo stabile nella realtà culturale e sociale napoletana. Sulla Biblioteca cfr. D’Alos-Moner; De Marinis, 1969, I, 225-227.

${ }^{12} \mathrm{Il}$ passo è riportato in Bentley, 69. Si veda anche Raina.
} 
Coronatae, adhibitis etiam praestantissimis matronis; quod ab Alfonso summo cum splendore servatum meminimus. Mirum, quam haec res nobilium animus conciliaret! quin etiam popularium, cum ex eius convivii apparatu reliquiisque liberalissime distributis urbs ipsa saturaretur. Quae quidem consuetudo, perinde ut alia quaedam digna exempla, omnimo est intermissa. His igitur in conviviis, sive, ut verius dicam, epulationibus, quoniam multitudo quam potest voluptate afficienda est, adhibendi etiam sunt qui artes tractent ludicras, utpote condimentu, quoddam epulationis; venter enim plenus, uti dici solet, tum videre, tum audire aliquid cupit, quod oblectet, et sensus animumque deliniat.

Trad. Fu pure una usanza introdotta dai re di Napoli, quella di imbandire ogni anno, in alcuni giorni stabiliti, un banchetto presso la chiesa di Maria Incoronata, con la partecipazione anche di nobilissime dame. Ricordo che da Alfonso questa usanza fu osservata con immenso splendore. È incredibile come questa iniziativa riuscisse a guadagnare l'animo della nobiltà e anche quello del popolo, giacché tutta la città si saziava con i resti del banchetto, distribuiti con grande generosità. Questa consuetudine, come altre che sarebbe bene portare ad esempio, è stata per sempre interrotta. In questi conviti, dunque, o banchetti, per dire meglio, poiché la gente deve essere intrattenuta nel modo più piacevole possibile, bisogna anche impiegare gli artisti dello spettacolo, quasi un condimento del banchetto: a pancia piena, come si suol dire, si desidera vedere o udire qualcosa che diletti i sensi e allieti lo spirito. ${ }^{13}$

La celebrazione o il ricordo della magnificenza delle feste aragonesi è parte significativa della retorica umanistica a Napoli. Questa si ritrova ad esempio anche nell'Arcadia del Sannazaro (edita dal Summonte nel 1504) lungo tutto il poema e con maggiore evidenza nelle prose XI e XII aggiunte alla prima versione del Libro

\footnotetext{
13 Pontano, "De Conviventia," 257-258. Il passo è trascritto in Addesso 2012, 11. Sulla magnanimità e l'investimento delle ricchezze del regno da parte del Magnanimo per dare splendore alla sua corte, rimane anche un ricordo di Borso d'Este che, giunto a Napoli, notava che il Magnanimo spendeva circa 1000 ducati che poi aumentavano maggiormente quando il re lasciava Napoli con il suo seguito. La nota è presa da Delle Donne, 255-270.
} 
pastorale nominato Arcadio (composto tra il 1486-1502). ${ }^{14}$ Ma altri riferimenti possono trovarsi in altre scritture letterarie, che contribuiscono a creare, come analizza in dettaglio Galasso, l'immagine di una "Napoli gentile e nobile:"15 dal panegirico di Angelo Catone (1474) anteposto al Liber pandectarum di Matteo Silvatico, dedicato al Re Ferdinando (Ferrante) I D'Aragona (1431-1494); o dalla lettera In laudem Neapolitane civitatis et Ferdinandi regis (1476) del fiorentino Francesco Bandini, al De Maiestate di Giuniano Maio (1492), fino alle più tarde Stanze sovra la bellezza di Napoli di Ioan Berardino Fuscano (1531) — edite di recente da Cristiana Anna Addesso ${ }^{16}$ —, che nel ricordo di una Napoli Aragonese in festa con giochi, pompe, giostre, riferiscono anche della memoria di 'voci poetiche,' canti, lire e musiche, celebrando i poeti dell'accademia pontaniana i loro studi e gli "ampi onori:"

Et si la nobiltà di cose nostre, si le faustose pompe et le bellezze, si gli lavori vorai che ti mostre, con gli ornati costumi et gentilezze, il valor d'arme, di cavalli et giostre, et l'armonia che avanzan le dolcezze di cimbali, de voci, organi et lire, più materia che tempo avrai da dire. (Canto I, ottava 96)

Lo sfondo su cui va letta la produzione poetica e recitativa durante il sessantennio del regno degli Aragona è dunque quello dell'intrattenimento elitario, dei fasti culturali dell'Accademia Pontaniana e delle valenze politiche e diplomatiche dei cerimoniali cortigiani, entro i quali si definisce il ruolo artistico e sociale del poeta aragonese, cantore/attore/accademico/diplomatico e ancora cerimoniere di corte durante le pubbliche feste, i banchetti e i conviti privati. Accanto a maestri riconosciuti quali il Panormita o il Pontano, Iacopo Sannazaro, e

\footnotetext{
${ }^{14}$ Per i profili diacronici del testo e la relativa tradizione manoscritta, oltre che per le prime edizioni, si veda Villani 1989, 75-108; Villani 2012. Cfr. anche l'ancora fondamentale studio di Corti, in particolare p. 288. Del rapporto tra lo spettacolo e la poesia recitativa aragonese e l'Arcadia mi sono di recente occupata nel saggio Arcadia, festa e performance alla corte dei Re d'Aragona (1442-1503), in corso di stampa.

${ }^{15}$ Galasso 1998.

${ }^{16}$ Fuscano, Stanze sovra la bellezza di Napoli.
} 
ancora il Cariteo e il cantore aquilano Serafino Ciminelli, operavano altri poeti d'indubbia rinomanza come Giovan Francesco Caracciolo, Francesco Galeota, Giovan Francesco Petrucci, Giuliano Perleoni, noto anche come Rustico Romano. Questi poeti, autori di Canzonieri sul modello petrarchesco, collaborarono con musici di mestiere e compositori, cimentandosi nella sperimentazione di quei generi - sonetti, canzoni, frottole, strambotti - tradizionalmente considerati come poesia per musica (o in musica), ${ }^{17}$ e inclusero nelle loro raccolte anche gli esiti del loro cimento con l'egloga in volgare, con la farsa, con l'autoctono genere dello gliommero o con la commedia. ${ }^{18}$

Oltre alle note del Pontano è possibile rintracciare tra le pagine di cronisti, commentatori o nei testi letterari stessi, interessanti testimonianze di questa prolifica collaborazione tra poeti d'eccellenza, improvvisatori e musici di mestiere. Le Effemeridi di Ioampiero Leostello, ${ }^{19}$ un diario giornaliero sulla vita del Duca Alfonso fra il 1485 e il 1491, riportano ad esempio di suoni, canti, letture e rappresentazioni che sembrano cadenzare la giornata allietando il Duca e la reale compagnia "innanzi facesse collatione" (14 dicembre 1488); o per l'arrivo del Re nella residenza prendendo "piacere de soni e canti" (2 giugno 1489); o ancora per occasionali conviti che rallegravano "tutto quello giorno [...] in festa cum danza et soni [...] et canti" (21 giugno 1499).

Il cronista non si sofferma sui tipi di canti poetici o sulle recite da lui notate, ma riporta interessanti annotazioni sui protagonisti di questi informali rituali poetici e canori. Racconta ad esempio che Francesco Galeota, nobile poeta e ambasciatore apprezzato della corte d'Aragona, fu cerimoniere abituale e intrattenitore del duca. ${ }^{20}$ Sempre il Leostello riferisce, tra le pagine delle Effemeridi, di componimenti recitativi denominati "farse et feste" o "danze, soni e [ancora] farse" che furono allestite tra il 1488 e il 1490 a diletto dei Reali d'Aragona, sia nelle occasione private ma anche in quelle ufficiali di matrimoni o per celebrazioni

\footnotetext{
${ }^{17}$ Sulla tradizione petrarchesca in genere nel XVI secolo si veda Richardson.

${ }^{18}$ Il termine 'commedia' presente in alcuni di questi Canzonieri quattrocenteschi si riferisce a componimenti che non avevano molto in comune con il genere comico classico, corrispondendo invece più probabilmente alla definizione di 'cantilenas morales' o più in generale ancora di componimento dialogato in volgare dai forti contenuti morali e civici.

${ }^{19}$ Leostello 1883. Una versione digitale è consultabile in rete nel sito Europeana: http://www. europeana.eu/portal/record/9200369/BibliographicResource_3000113670409.html

${ }^{20}$ Flamini 1892. Cfr. anche Santagata, 370; e Parenti 1979, 178n.
} 
civiche. Racconta, allora, di un Sannazaro attore, impegnato insieme al Cariteo, poeta improvvisatore e anch'egli funzionario degli Aragona, nella recita di "certe farse" per alleviare la convalescenza del Duca a Castel Capuano:

\section{Die xxj Augusti}

Bona hora comedit et audita missa expedivit quedam negocia cum Secretario Regio: et quam primum dormivit per duas horas. Et tucto quello jorno stecte bene senza febre. Et hoc intellecto Rege venne sua maesta cum Regina a visitarlo hora xxiij. Et stecteno in piacere circa una hora et disceserunt assai allegri vedendo S. Duca gia sano. Et quam primum cenavit et hora iij. quievit. Et eo sero vennero certe farse fra le quali fu Jacobo Senazara et cariteo: et de cio lo I.S. prese grande recreatione et piacere.

I due poeti, Sannazaro e Cariteo, sono inoltre ricordati ancora insieme nel noto commento cinquecentesco alla canzone Io canto in discanto, in occasione questa volta dei festeggiamenti sontuosi del 1496 per le nozze di Ferdinando II d'Aragona, detto Ferrandino (1467-1496). Entrambi sono direttamente coinvolti nella messinscena dei loro versi, nella veste di 'registi' o 'ordinatori' invece che attori, questa volta. Per l'occasione furono, infatti, allestiti molti intermezzi, farse, egloghe, e al Sannazaro è dato il compito insieme all'illustre Giovanni Pontano di far recitare i loro gliommeri napoletani; mentre il Cariteo fece cantare alcune delle sue frottole tratte dal suo Endimione.

Hor questa sì universal allegrezza, oltre il danzare, e banchetteggiare non vi mancorno mille sollazzevoli intermedi de musiche d'ogni sorte di farse, d'egloghe, ch'ivi s'intesero; et il Pontano, et il Sannazaro, che ivi erano ferno recitare non sò quanti di quelli loro gliommeri napolitaneschi, et Carideo, che Barcinio è chiamato dal sannazzaro nell'Arcadia, essendo costui segretario del Rè fe cantare mille sue frottole, fatte da lui in lode della sua luna, di cui egli sotto il nome d'endimione era mirabilmente invaghito[....$^{21}$

\footnotetext{
${ }^{21}$ Il passo è in Addesso 2009, 14-15. Il manoscritto che contiene la canzone è conservato presso la Società Napoletana di Storia Patria.
} 
Il cronista si riferisce al Cariteo ricordando anche il suo nome pastorale di Barcinio, protagonista dell'egloga XII dell'Arcadia sannazariana, quasi a voler fissare attraverso la 'maschera' pastorale creatagli dal Sannazaro nel suo scritto l'identità artistica del poeta sulla scena teatrale moderna a eterna memoria. La dimensione pastorale è testimoniata dall'anonimo cronista anche nella descrizione della canzone rappresentata al termine dell'intera giornata festiva, intonata da due cavalieri, l'uno in abito di pastore accompagnandosi con la sordellina (strumento d'invenzione napoletana simile alla cornamusa o zampogna) e l'altro vestito alla cortigiana e accompagnato da una lira.

[...] et all'hora il Rè voleva andare in letto comparve una mascherata d'alquanti cavallieri riccamente addobbati, fra i quali vi furono due l'uno vestito da contadino sonando una sordellina, e l'altro alla corteggiana sonando una lira, i' quali dopò haver sonato un pezzo concordemente cantaro questa canzone il cui intendimento voi signora desiate sapere, la quale porse non poco piacere à gl'ascoltanti, e vi fur di quelli, che vedendo l'hora che fu cantata la giudicaro per un epitalamio villanesco, ma in esso non vidde quello artificio, che in quel si cantò nelle nozze di Peleo, e di Theti, finalmente la canzone diede da ridere al Rè, et alla Regina, et à suoi corteggiani per la vaga ruzzezza delle parole di che era tessuta, benche il vero senzo di lei non fusse inteso da veruno fuorche dal Sannazaro, e dal Pontano, e dal Caracciolo. ${ }^{22}$

Richiamando alla mente di alcuni più scaltri spettatori la mitica competizione musicale tra zampogna e cetra, i due musici-cantori intonano le rispettive canzoni (prima in contrasto poi 'congiuntamente'), che sembrano piacere molto alla nobile comitiva per il linguaggio rustico e villanesco che suscitò tra il pubblico grande ilarità. Tuttavia, avvisa il cronista, non tutti videro l'artificio dei due artisti che in realtà, entro la composizione 'villanesca,' rappresentarono il mito di Paride; e non tutti colsero il "vero senzo" della canzone, fatta eccezione per il Sannazaro, il Pontano e il Caracciolo, che nascondeva richiami e riferimenti probabilmente politici, con possibili toni anche di denuncia e critica, come il genere prevedeva e come molta poesia pastorale scritta e recitata nel regno celava attraverso l'allegorismo e la maschera bucolica. Come osservato da Pieri, "molti di

\footnotetext{
${ }^{22}$ Ibidem.
} 
questi poeti, del resto, rivivono sulla propria pelle conflitti di un regime signorile analoghi a quelli da cui era scaturito il grande libro virgiliano: dalle confische dei beni alle gelosie cortigiane." 23

Registriamo dunque attraverso le parole del commentatore cinquecentesco una compresenza dell'elemento mitico e del linguaggio rustico, comico, apparentemente popolare, che appare finalizzata all'elaborazione di una drammaturgia se vogliamo elementare ma che condensava una duplice (o forse triplice) valenza scenica, letteraria e comunicativa. In altri termini, osserviamo la messinscena da parte di una cultura elitaria, quella dei poeti umanisti, di uno spettacolo gradevole per la comitiva regale, grazie all'intervento di un linguaggio espressivo popolareggiante che facilmente suscitava il riso fra gli spettatori, ma che al tempo stesso consentiva la trasmissione di un messaggio preciso, tipizzato - apprendiamo dal cronista - attraverso una sottile trama di riferimenti eruditi che erano evidentemente percepibili solo a pochi. I poeti umanisti napoletani avevano, infatti, elaborato per l'occasione un linguaggio scenico differenziato a cui affidarono il loro messaggio politico, accostando al mimetismo linguistico di matrice popolareggiante il richiamo al mito e all'immaginario pastorale con l'ausilio di moduli teatrali di musica, danza e canto come divertimento colto del pubblico regale, nobilitando così le pratiche dello spettacolo e contribuendo alla elaborazione - sul modello probabilmente fiorentino del circolo dei poeti medicei — di una cultura elitaria distinta e intimamente legata alla famiglia reale e ai luoghi del potere.

Effusività bucolica ed espressività pseudo-dialettale si contrappongono anche nei tre moduli (o monologhi) che compongono il superstite gliommero del Sannazaro Licinio, se' l mio ingegno fusse ancora. Ciò si osserva nei "bruschi chiaroscuri — come commenta Pieri — fra il registro del rimpianto amoroso vagamente arcadico e il minuto cronachismo gastronomico-cittadinesco della parte dialettale in cui sfilano fatterelli e ritratti del suburbio partenopeo." ${ }^{24} \mathrm{E}$ ancora, temi bucolici e temi popolari, si confrontano nello gliommero Eo non agio figli né fittigli — ritenuto originariamente del De Jennaro e ora attribuito da De Blasi al Sannazaro stesso - dove ritorna il topos bucolico del lamento nostalgico dei tempi passati dai toni tuttavia villaneschi e popolareggianti che ritroveremo anche nelle egloghe dei Rozzi a Siena, poi in Ruzante e ancora in tanti zanni dell'Arte. ${ }^{25}$

\footnotetext{
${ }^{23}$ Pieri, "Dalla lirica alla festa," $81-82$.

${ }^{24}$ Pieri, “'Sumptuosissime pompe," 61.

${ }^{25}$ Ibidem.
} 
È possibile credere che l'esibizione "in forma di pastore" rientrasse in una pratica abituale di recitazioni sotto il velame pastorale. Una prassi ispirata dalle occasioni contingenti dei cerimoniali di corte e contraddistinta da riferimenti alle vicende politiche locali e di tipo autobiografico, sia pure filtrate dall'allegorismo bucolico. Ne abbiamo conferma, ad esempio, nella Canzone recitata, per l'appunto in abito di pastore, dal poeta romano Giuliano Perleoni (il Rustico), in onore del duca di Calabria per i festeggiamenti della presa di Otranto nel $1482 .{ }^{26}$ Sappiamo inoltre che il Rustico si era già cimentato con il genere bucolico componendo sonetti pastorali con il senese Filenio Gallo. Sempre del Rustico è l'egloga di Thelemo e Phylomeme scritta (o forse riadattata da un testo originario) in memoria della morte di Galeazzo Maria Visconti, e raccolta insieme a un Tryumpho del duca di Milano nel Compendio.

La prassi attorica ancora prescinde, in questa fase di sperimentazione, dalle categorie di genere, di là da venire. Lo sguardo è primariamente rivolto al luogo e all'occasione della recitazione, contraddistinta da un linguaggio allegorico che stratificava la funzione della rappresentazione su diversi livelli e piani di ricezione. Questi orientavano le scelte drammaturgiche e la definizione di moduli e tecniche recitative, trascendendo, di volta in volta, modelli e repertori in funzione dell'invenzione attorica e di un sapere attoriale oltre che autorale. ${ }^{27}$

Si tratta di testi che letti in controluce, nella loro sostanzialità orale, ossia in una dimensione di oralità e dunque di presenza di voce e volto dell'attore, possono ancora rivelare i segreti delle loro tecniche espressive e narrative. Attraverso queste forme di poesia orale possiamo così definire il rapporto esistente tra l'oralità letterata e quella delle pratiche rappresentative.

Avviando un'analisi incrociata tra i profili biografici di questa fervida e variopinta cerchia dei poeti ambasciatori e cerimonieri aragonesi, e i dati dello spettacolo e testuali in nostro possesso, cercheremo di comprendere e acquisire alcuni degli elementi che concorrevano nel pensiero e nella pratica umanistica

\footnotetext{
${ }^{26}$ Il Rustico era entrato al servizio di Ferrante dopo la chiusura dell'Accademia Pontaniana. Canzone V de nuova textura al Illustrissimo S. Duca di Calabria recitata in un convito in forma d'un pastore in la recuperatione d'Hydronto, raccolta in: R. Romano, Compendio di sonetti et alter rime de varie texture intitolato il Perleone, Napoli, Aiolfo de Cantono, 1492. Cfr. Addesso 2012, 43-62; De Blasi 1993, 130.

${ }^{27}$ Sulle questioni che pertengono la sfera di situazioni recitative in senso lato e il rapporto tra oralità e scrittura si vedano Ong; Zumthor. Punti di riferimento nell'ambito degli studi teatrali sono: Ruffini; Ventrone 1993; Guarino 1995.
} 
alla creazione del modello del poeta moderno. Si tratta di delineare un profilo di artista complesso e articolato, che attraverso la lente dell'antico nel richiamo al mitico cantore Orfeo, e le tecniche recitative poetiche e musicali contemporanee, si presenta sul finire del Quattrocento prima di tutto come performer: per la sua capacità oratoria, il decoro, l'abilità di raggiungere una perfetta armonia tra voce, gesto e ritmo, la perizia nella rielaborazione testuale in funzione del momento esecutivo, improvvisato o predeterminato, e ancora le attitudini linguistiche e dicitorie.

Da questa prospettiva, i testi assumono una nuova luce divenendo essi stessi documenti inestimabili per identificare i processi creativi che pervengono non solo all'universo della scrittura ma anche a quello della recitazione e consentono perciò di evidenziare l'apporto specifico del poeta-performer alla costruzione di componenti recitabili della poesia orale e del ruolo da essa assunto nella società cortigiana, e in quella aragonese in particolare.

La forma scritta del testo verrebbe ad essere la semplice registrazione di uno spettacolo allestito durante un'occasione festiva. ${ }^{28}$ Oppure il testo potrebbe essere il risultato di un'operazione di confezione artificiale di porzioni di testi che erano nati in tempi e modi diversi, come fu probabilmente il caso dell'omaggio del Perleoni alla memoria del duca di Milano; o ancora invenzione letteraria di situazioni rappresentative dentro il testo (recitativo o no) che mirava a riproporre circostanze 'tipo' della recitazione estemporanea e di pratiche dicitorie. Questo modo di procedere è ad esempio presente in alcune farse, come vedremo nel dettaglio, ed è ricorrente, come ho già avuto modo in più occasioni di argomentare, per il genere egloghistico e pastorale per il quale il Sannazaro "in quelli tempi otteneva la palma:"29 per la struttura del canto amebeo che riproduce le convenzioni della poesia orale e del canto monodico alla lira caro alla pratica umanistica; per l'attivazione di una mitologia modernizzata e l'uso del linguaggio allegorico entro il quale si riflette la società contemporanea; e per il costante richiamo a circostanze dilettevoli che radunavano tanto nella finzione letteraria quanto nei luoghi reali della festa aragonese un numero selezionato di persone (pastori-poeti e ninfe-cortigiane) per allietarsi in feste e dolci canti, ricreando in questo modo in chi ascoltava questi testi l'immediatezza dell'azione agita hic et nunc propria della

\footnotetext{
${ }^{28}$ Come, ad esempio, nel caso della descrizione che Iacopo Sannazaro lasciò delle sue due farse fatte rappresentare nel 1492 a Castel Capuano per i festeggiamenti della presa di Granata.

29 "Vita del facondo poeta vulgare Serafino Aquilano per Vincenzo Calmeta composta," in Calmeta, 65 .
} 
comunicazione orale. ${ }^{30}$ La tensione tra letterato e 'maschera' del pastore arcade, o del 'costume' del villano/popolano (nel caso degli gliommeri e di alcune farse), e ancora il richiamo alle figure allegoriche e mitologiche, divengono, entro la cornice evasiva e politica dello spettacolo e dei cerimoniali di corte, un fatto teatrale. $\grave{E}$ dunque nella dimensione dei pubblici uffici, delle occasioni d'incontro politico e dell'intrattenimento elitario che a Napoli vanno cercate le modalità della comparsa di forme del dire che si traducono nella festa cortigiana in formati più specifici connessi al teatro a partire dalla progressiva emersione di un volgare illustre napoletano e di meccanismi di comunicazione poetica e politica che la poesia orale a corte attivava nella definizione di una nuova drammaturgia per la scena cortigiana.

\section{Oratoria. Il poeta ambasciatore: dicitori del potere}

Di aspetto non molto gradevole - secondo quanto lasciò registrato Pietro Cennini nei suoi Ricordi —, basso di statura e con un naso prominente, ${ }^{31}$ Antonio Beccadelli, detto il Panormita (1394-1471) è ricordato soprattutto per la popolarità e il consenso che riusciva a raccogliere tra gli uomini dotti. Il suo amico, poeta e ambasciatore genovese, Bartolomeo Facio o Fazio (1405-1457) — poi funzionario e storiografo napoletano - lo descrive come un uomo di vastissima memoria (che "tiene fisse nella memoria tutte le storie antiche") e di lui ricorda l'indefessa opera di trascrittore di codici che portava con sé anche durante i

\footnotetext{
${ }^{30}$ Bortoletti, Egloga e spettacolo; Ead. "Per una nuova drammaturgia. L'egloga nel Quattrocento italiano," pp. 67-108; Ead. “Arcadia, festa e performance." Sulla sfera delle situazioni recitative in senso lato, le pratiche dicitorie e recitative, e sul rapporto tra testo scritto e testo recitato si rimanda al fondamentale studio in area fiorentina con specifico riferimento alla tradizione novellistica di Ventrone 1993. Come nota la studiosa, "d'altro canto quello della circostanza conviviale ed esclusiva posta come conditio ricorrente, se non addirittura necessaria, degli intrattenimenti poetici e fabulistici, si era ormai stabilito come topos di lunga durata a Firenze e altrove, a partire dal modello decameroniano, passando per il Paradiso degli Alberti, fino alle Porrenttane di Sabbadino degli Arienti e agli Asolani del Bembo, per richiamare solo qualche esempio" (92). Si veda anche il recente contributo Ventrone "'Civic Performance' in Renaissance Florence" (in corso di stampa).

31 " [...] grande nella persona, ma d'aspetto non molto aggradevole: ebbe il naso, che si andava assottigliando verso le ciglia, e s'ingrossava verso le narici: e che inoltre essendo ancora corto, e piccolo, contribuiva anche a renderne non felice la fisionomia." Si cita da: Colangelo. Quanto a Pietro Cennini, egli era un prolifico trascrittore di codici tra cui anche il De dictis et factis Alphonsi dello stesso Panormita.
} 
suoi numerosi viaggi e persino in battaglia a fianco del Re. Ancora, riferisce del dono di "un bell'ingegno" e del "suo valore nella poesia, come nella prosa: il che rarissime volte si ottiene.” Al Panormita è attribuito anche dal Pontano il merito di aver "restituita quasi nell'antica forma la poesia non pur moribonda, ma quasi dimenticata nell'Italia. ${ }^{32}$ In lode del suo maestro, il Pontano commemorava la dolce musica della sua lira che "con la voce accompagnava delicati suoni," lasciando di lui un ricordo poetico riccamente sonoro, in cui invitava i passanti a fermarsi al suo sepolcro e a udire il canto delle Muse, delle Sirene e della natura tutta, esortandoli ad unirsi a loro per celebrare i canti del sommo poeta dandone eterna memoria.

Conveniunt nunc ad tumulum, celebrantque choreas

et memorant lusus, magne Poeta, tuos.

En audis, sonet ut lenis concentibus aura?

Ut sonet appulsu concita terra pedum?

Haec vatis memores Musae post fata rependunt:

carminis hoc meritum est. Num salis? Hospes abi. ${ }^{33}$

Trad. Or sulla tomba le Muse s'adunano e celebrano danze / e ricordano i tuoi giocosi carmi. / Non senti tu come dolce di musiche l'aria risuona? / Come risuona il suolo al battere dei piedi? / Così le Muse ripagano, grate, l'estinto poeta: / questo il merito dei carmi. Non basta? Ora va, passante.

Ma più di tutto, del Panormita è ricordato ancora dal Facio che "fu stimato eloquentissimo nel persuadere, o nel dissuadere" ed "ebbe una penetrantissima forza d'intendimento, unita alle grazie, ed alle liberali facezie," 34 creando un'immagine di eccellenza del suo amico poeta e oratore per la capacità di riscuotere l'applauso dei suoi auditori ma soprattutto, come oratore, di saperli persuadere. Questo profilo sarà ripreso anche dal Pontano nei Dialoghi e riadattato sul modello ciceroniano che egli coniugherà con quello quintilianeo in un originale equilibrio

\footnotetext{
${ }^{32}$ Pontano, De Sermone, I. 6. t. 2. p. 247

${ }^{33}$ Pontano, Tumuli, Liber primus, XX trascritti in Vita di Antonio Beccadelli.

${ }^{34}$ Facio, De Rebus Gestis Alphonsi Regis. Si trascrive sempre da Vita.
} 
tra oratoria, storia e poesia, riflettendo sul destino dell'antichità nei tempi moderni e delineando la figura del nuovo saggio. ${ }^{35}$

Nato a Palermo, sebbene di famiglia bolognese, il Panormita fu insegnante di retorica a Pavia (1430-1431), incoronato poeta a Parma (1432) e, fatto ritorno nel Regno, fu sovraintendente della biblioteca reale, amministratore della dogana regia di Palermo e infine ambasciatore d'importanti missioni diplomatiche, consacrando il suo tempo ai doveri ufficiali, mentre in quegli stessi anni creava a Napoli la nuova Accademia - la futura Pontaniana. ${ }^{36}$ Lo troviamo protagonista sin dalle delicate fasi di conquista del Regno di Alfonso, durante l'assedio a Gaeta, inviato dal futuro re a esortare la città alla resa; quindi a Firenze e a Siena nel '36 a recitare una sua orazione per scoraggiare una potenziale alleanza fra le due città con Venezia e Genova contro Filippo Maria Visconti, amico fedele degli Aragona. È poi ambasciatore a Venezia, per chiedere - secondo almeno una leggenda - un braccio dello storico Tito Livio di cui si era ritrovato il corpo in quegli anni, ma più verosimilmente per stringere l'alleanza con la Repubblica. E ancora, nei primi mesi del regno di Ferrante, il Panormita recita a Milano la sua orazione al Duca Francesco Sforza per ringraziarlo, a nome del suo re, di aver appoggiato il regno contro papa Callisto III; mentre l'anno dopo si rivolge a Pio II per guadagnare consensi sulla politica anti-genovese di Ferrante. Le sue orazioni furono frequenti anche negli eventi festivi in ambito aragonese, come accadde per i sontuosi cerimoniali del 1452 in onore del passaggio del Re Federico III di Germania, a cui il Panormita indirizzò la sua recita oratoria unitamente a quella di Andrea Partenopeo (forse il fiorentino Alemanni). ${ }^{37}$ La nomea delle sue orazioni fu tale che, secondo un racconto del suo biografo Francesco Colangelo, a Verona il podestà, i pretori e altri signori della corte applaudirono, acclamarono e onorano con lauti banchetti "un tal uomo" calabrese o siciliano che ebbe l'audacia

\footnotetext{
35 Del Pontano, si pensi alla parte conclusiva dello Charon, all'iniziale elogio sul Panormita nell'Antonius e ancora all'Actius dove, come nota Francesco Tateo, e come vedremo a breve, diviene centrale la figura della Poesia. Tateo, 68-132. Si veda anche Deramaix.

${ }^{36}$ Per una biografia dettagliata sul Panormita cfr. Natale; Ryder 1976; Santoro.

37 Sull'orazione di Andrea Partenopeo e la sua identificazione si veda De Marinis e Perosa, 97-104. Sui festeggiamenti per Federico III e le sue nozze con Eleonora di Portogallo, cugina del Magnanimo, scrissero sia il Pontano, il Facio che il Panormita e Andrea Partenopeo. Un interessante ricordo è lasciato anche dalle Memorie del Duca di Ossuna.
} 
di spacciarsi per il Panormita recitando un'orazione parte in versi e parte in prosa per la nobile comitiva. ${ }^{38}$

Come il Panormita, gli umanisti e poeti aragonesi accettarono uffici politici ricoprendo funzioni di spicco in tutte le vicende cruciali dei Re: dalla conquista del regno, per l'appunto, alle lotte per la successione di Ferrante, alle rivolte dei baroni fino alle invasioni straniere. Fra gli incarichi dei poeti aragonesi vi era, infatti, quello di rappresentare come per il Panormita il governo del re nelle ambascerie e ancora nelle cerimonie ufficiali sia all'estero sia in città, ma anche di svolgere funzioni giuridiche o amministrative, e di recitare a diletto del principe o ancora nelle feste pubbliche.

Questi dotti ed eruditi poeti ricoprivano dunque sia mansioni ufficiali e pubbliche, sia incarichi legati alla routine della corte e, in entrambi i casi, le abilità richieste per l'assolvimento di tali uffici erano di tipo letterario e recitativo. Dovevano cioè essere in grado di comporre e improvvisare versi su argomenti vari — morale, storico, gnomico, encomiastico — , costruendosi un repertorio ricco e diversificato da recitare nelle diverse occasioni di palazzo, di ricreazione o di incontro politico, mostrando la perfetta padronanza tecnica vocale, e abilità sia mimiche, sia mnemoniche. Nelle missioni diplomatiche la ricezione del messaggio che il sovrano inviava e il buon esito della trasferta erano dipendenti non solo dal significato della missiva ma anche dal modo in cui veniva presentata. Come nota Paola Ventrone per gli araldi fiorentini, "era quindi necessario che il portavoce, al momento della comunicazione, [...] assimilasse [il significato dei messaggi] prontamente e alla lettera, e ne sapesse restituire, una volta ritornato [...], anche i modi di espressione." 39

A Napoli, l'Accademia del Panormita, la futura Pontaniana, era luogo di aggregazione di questi eloquenti poeti entro cui si stringeva e articolava il rapporto clientelare con la corte aragonese. Era una relazione complessa, fatta di dedizione e rappresentanza verso i sovrani, di cui ci si faceva interpreti e divulgatori delle loro rispettive idee e strategie attraverso la scrittura ma anche attraverso le arti oratorie e recitative, l'eloquenza e l'abilità nel persuadere l'auditorium con arguzia e facezia (come nel modello del Panormita fissato dal Facio e dal Pontano). Ciò

\footnotetext{
${ }^{38}$ Colangelo, 75.

${ }^{39}$ Ventrone 1993, 139. Il poeta aragonese sembra assumere le medesime mansioni dell'araldo fiorentino ("sindicus et referendarius") e insieme anche quelle che sempre a Firenze ricopriva la funzione di "cavaliero di palagio."
} 
rivela l'identificazione dell'arte dell'intellettuale-letterato con la abilità dicitoria e, nel caso specifico dell'oratoria politica, e dei portavoce. ${ }^{40}$

Il riconoscimento di una teatralità propria di questo genere di componimenti e recite come le orazioni (o, in altri ambiti, le prediche), legati all'espressione orale prendeva le mosse da un'idea convenzionale di 'teatro medievale' e dall'attività di oratori (o predicatori) recitanti nelle pubbliche piazze che, come osserva Ventrone, esercitavano un'arte che può avvicinarsi con buona approssimazione a quella dell'attore di professione. ${ }^{41}$ Ma nella cultura umanistica e nelle fucine dell'accademia e della corte essa acquista il senso di una complessità operativa e teorica che elabora progressivamente valori e modelli di una cultura elitaria fondati sulla perizia espressiva e su una lucida coscienza della differenza esistente tra un linguaggio parlato e un linguaggio scritto, mostrando la volontà di scegliere, a seconda dei contesti e delle situazioni specifiche (ossia della cornice ora ricreativa, ora celebrativa, ora della trattativa politica), l'immediatezza dell'azione performativa. Non era dunque evento straordinario la performance ad esempio di un "Maso Antonio Ranzone," ambasciatore del duca di Calabria, nei panni della Virtù ("in abito leggiadrissimo e severo") per la Rappresentazione allegorica della Voluttà, Virtù e Fama ${ }^{42}$ messa in scena da Serafino Aquilano, e da lui recitata nel ruolo della Voluttà, alla corte di Mantova nel 1495. Per l'occasione il Ranzoni cantò, in endecasillabi con rimalmezzo, le lodi della Virtù e insieme il deploro "per il disprezzo che in essa era di questi tempi tenuta" fatta eccezione per i due destinatari della recita - il duca di Calabria e il marchese di Mantova, omaggiati in chiusura della rappresentazione dai versi recitati dalla Fama.

I ricordi sulle missioni e recite di questi poeti-ambasciatori, e all'occasione performer, della monarchia aragonese si potrebbero arricchire attraverso la ricerca

\footnotetext{
40 Sull'assunzione da parte della cultura umanistica, e del Pontano in particolare, dell'arte dell'eloquenza come arte della parola per eccellenza, in ripresa del pensiero ciceroniano e quintilianeo, e della relazione tra questa arte, la poesia e la storia, si rimanda ancora a Tateo, 74-78.

${ }^{41}$ Ventrone 1993, 94; Bolzoni, e ivi la bibliografia.

${ }^{42}$ Il testo fu pubblicato da Pietro Ferrato. Cfr. Ciminelli (Aquilano), Rappresentazione allegorica data in Mantova nel 1495. Stampata solo in 50 esemplari l'edizione si basa su una lettera scoperta dal can. Willelmo Braghirolli e conservata nell'Archivio di Stato di Mantova (F. II.6, busta 2110). Fu poi ristampata più volte, tra cui dal Torraca, dal Menghini e ora da Rossi: Ciminelli (Aquilano) 2005, 423-435. Per l'elenco completo delle edizioni della rappresentazione cfr. Giustiniani, 102.
} 
di altre note tratte da altra corrispondenza di lettere, dalla trattatistica e ancora in altri ricordi poetici, a verifica della familiarità di queste figure di intellettuali con un sistema di relazione che si muoveva all'interno dei circuiti di comunicazione orale, e che trovava un'eco anche nelle scritture di opere recitative, influenzandone in alcuni casi le scelte più fortemente drammaturgiche. ${ }^{43}$

Di queste forme di comunicazioni riportate o 'ripresentate' legate all'ambascerie troviamo ad esempio una testimonianza letteraria e teatrale in una delle farse del Caracciolo (Pier Antonio), che egli recita — come si evince dal titolo — nei panni di un araldo ("sotto vestigio di ciaraldo") di fronte al Duca di Calabria, Alfonso I, facendosi ambasciatore, nella finzione teatrale e nella realtà della cornice della festa, di un messaggio per la corte. ${ }^{44}$ Ma ne abbiamo traccia anche in una delle sei farse del Sannazaro di cui ci sono pervenuti i testi e, in alcuni casi, qualche preziosa nota sulla avvenuta rappresentazione. Si tratta della farsa dell' Ambasciaria del Soldano esplicata per lo interprete, rappresentata alla corte aragonese nel $1490 .{ }^{45}$ Nell'economia di questo primo quadro investigativo, la farsa del Sannazaro ci interessa per la dimensione di oralità e delle situazioni recitative in senso lato che il Sannazaro riproduce in scrittura rappresentativa nella forma di un monologo 'dialogato.' Scritta per una sola voce recitante (in endecasillabi con rimalmezzo), la farsa dell'Ambasciaria rappresenta la recita da parte di un messaggero delle parole d'amore del suo Soldano di fronte alla donna da lui amata, e la consegna di tre doni come segno del suo amore devoto.

La farsa del Sannazaro ripropone dunque lo schema della comunicazione orale di un messaggio, che si legava in questo caso al tema amoroso, ma che riproponeva, nella dimensione festiva aragonese in cui la farsa era rappresentata, strategie comunicative familiari alla corte perché simili a quelle situazioni di ambasceria politica di cui furono, come abbiamo visto, protagonisti apprezzati un Panormita, un Facio o altri poeti umanisti aragonesi. Anche all'ambasciatore della nostra farsa, infatti, il sovrano affidava con estrema fiducia il suo messaggio (d'amore), contando sull'infallibile efficacia elocutoria e l'abilità persuasiva del suo interprete, sulla sua viva voce e sulla sua presenza di fronte al destinatario del messaggio (la

\footnotetext{
${ }^{43}$ Proseguendo la similitudine con l'araldo fiorentino cfr. Ventrone 1993, 125-126.

${ }^{44}$ Simile per struttura e tema è anche la farsa recitata dal Caracciolo nei panni del 'turcomanno' di fronte alla principessa Sanseverino di Bisignano, cui rifaremo ancora un cenno in chiusura del nostro excursus. Cfr. Napoli-Signorelli, vol. 3, 365; Addesso 2012, 17, 93-94; Nevola, 59-68.

${ }^{45}$ Croce 1891, II, 768 . Il testo è edito nell'edizione citata di Mauro.
} 
donna amata), affinché, superando anche le barriere linguistiche ("lo qual non sa il linguaggio italiano," v. 19) ${ }^{46}$ grazie al suo virtuosismo retorico, egli "[...] dica quel che non può dirse in carte" (v. 21).

Dice che queste ampolle

da le intime medolle e da le vene

son tutte quante piene e ben colmate

$[\ldots]$

Dice che ognora sente

una aspra fiamma ardente in mezo l'ama,

una angosciata salma et uno ardore

[...]

Diceme ch'io ve preghi

che per vui non se neghi omai risposta

degna de tal preposta qual portamo. ${ }^{47}$

Entro la struttura monologante, Sannazaro crea, come nota Bianchi, l'illusione di un andamento dialogante tra più personaggi (l'ambasciatore, la dama e il soldano) che rafforza la teatralizzazione del testo stesso, completata dal riferimento alla risposta della dama sempre attraverso le parole del mediatore:

Queste vostre parole

rare e nel mondo sole, alma mia Diva,

seran cagion che viva il signor mio.

Nella composizione di una 'virtuale' scena dialogata che l'ambasciatore riesce a creare, Sannazaro non manca di includere, in ripresa del codice bucolico, il

\footnotetext{
${ }^{46}$ Come nota Bianchi il riferimento alla lingua italiana si legava con buona probabilità "a una rappresentazione verosimile di quelle figure di interpreti e mediatori linguistici tra Oriente e Occidente, chiamati anche 'dragomanni' che mediavano pure traffici e affari di vario tipo." Bianchi, 64-65. Ma certamente non mancavano esempi di contraffazioni o 'adeguamenti' linguistici di questi 'mediatori' anche nei circuiti peninsulari per la ricchezza degli idiomi dialettali che diventarono spesso motivo di derisione nelle satire contro i villani, ma furono anche occasione e pretesto di virtuosismi espressivi nelle recite anche a corte di ('nobili') buffoni, attori e poeti in quanto specialisti della parola e della scena.

${ }^{47}$ Si trascrive sempre dall'edizione di Mauro.
} 
riferimento al mitico cantore tracio e ai poteri lenitivi del canto per le pene d'amore, che caricava il testo e la performance di rimandi classici d'immediata connotazione aulica comprensibile al pubblico, almeno fra gli spettatori più avvertiti. L'immagine del Soldano innamorato che l'ambasciatore crea, ripropone, infatti attraverso gli artefici del genere pastorale, una visione che è prima di tutto sonora e performativa: il suono del suo pianto di triste innamorato, dei suoi canti ("per sfogarse" e per attenuar il suo "foco"), delle sue grida (capaci di muover come nel mito orfico "tronchi e sassi"), della sua voce dolente, di cui l'ambasciatore si fa evidentemente interprete.

Infine l'omaggio dei doni — "le acque fine," i "pivetti odoriferi," e la polvere "di Cipro" - , che il messaggero mostra alla dama, rafforzano il 'monologo dialogante' nella sua dimensione visiva grazie a una serie di richiami a figure simboliche e potenziali situazioni sceniche, come il bruciare dei profumi e l'accensione della "amorosa fiamma" ("atti a mostrare quando fuma, / che così se consuma l'alma stanca," vv. 45-46, del Soldano e il suo ardore per la dama). Queste immagini dovevano essere probabilmente esibite, come ipotizza Bianchi, per divertire ed essere facilmente comprese dal pubblico di corte, che nella cornice festiva aragonese in cui la farsa sannazariana è rappresentata diviene esso stesso testimone del messaggio privato e destinatario dell'omaggio poetico e teatrale.

La figura dell'ambasciatore si tipicizzerà poi nella commedia cinquecentesca in quella del messaggero (specie d'amore) assumendo un ruolo sovente strategico per lo sviluppo drammaturgico della piece. Gli esempi possono essere numerosi, ma rimanendo tra i luoghi dello spettacolo aragonese, un messaggero appare, sia pure con un ruolo secondario, in una recitazione in forma, questa volta, di commedia nova ${ }^{48}$ ("misteriosa e colma, d'excellentia," come recita il nunzio Minerva) intitolata Gaudio d'amor, stampata a Venezia nel 1526, ma composta sul finire del Quattrocento per mano del celeberrimo quanto misterioso rimatore Notturno Napolitano. ${ }^{49}$

L'identità di questo prolifico poeta non è ancora chiara agli studiosi che lo hanno identificato ora con Antonio Epicuro, ora con Pier Antonio Caracciolo,

\footnotetext{
48 Laggettivo "nova" accompagna l'edizione anche della sua raccolta di sonetti, strambotti, canzoni, egloghe, che ebbe larga fortuna nel corso del cinquecento insieme al Fior de Delia dell'altro poeta napoletano apprezzato dall'editoria veneziana quale fu Antonio Ricco. La raccolta segue, come notava Rossi, la 'discendenza' dei canzonieri di un Tebaldeo e di un Serafino. Rossi, 811. Su Notturno napoletano cfr. Torraca, "Pier Antonio Caracciolo," 65-81.

${ }^{49}$ Notturno, Comedia noua de Notturno Napolitano intitolata Gaudio d'amore.
} 
seguendo l'indicazione di alcuni cataloghi che associarono questo nome alla ricca produzione del Notturno. ${ }^{50}$ In questa nuova commedia, a metà tra la composizione cortigiana e la futura commedia regolata, costruita intorno al tema delle strategie d'amore, il messaggero è in realtà una messaggera: Philotea, custode — secondo il racconto della ruffiana Scaltra - del messaggio d'amore di un nobile gentiluomo invaghito della Madama Provida, rimasta vedova e ora in cerca di 'un buon partito':

Tutto il giorno ambasciate ho d'ogni canto

Non di persone vil, ma d'homin degni

Da portarne alfin gloria, utile, e vanto,

El bisogna mo, Scaltra che t'ingegni

A pensar quel che in ciò debbiamo fare

E che a tuo modo, mi amaestri, e insegni.

Questi messaggi d'amore, recitati fedelmente da ambasciatori 'degni,' si fanno sovente portatori, nell'economia della commedia, di valori morali funzionali al buon regolamento sociale che, sia pure in forma nuova, si legavano in parte a quei precetti dell'oratoria nuziale umanistica che, a Napoli, trovavano espressione sia nella trattatistica pontaniana - sono gli anni del De Obedentia (1472) e del De amore coniugali (1461-1486) - ma naturalmente anche nell'oratoria dello spettacolo cortigiano dei banchetti nuziali.

Nel 1473, ad esempio, per i noti e sfarzosi festeggiamenti delle nozze tra Eleonora d'Aragona ed Ercole I d'Este, ${ }^{51}$ il Pontano stesso compone un'orazione nuziale, intitolata l'Epithalamium Leonorae Aragonie, recitata insieme a quella di Giovanni Brancati e pronunciata con grandi lodi a celebrazione della sacra unione matrimoniale, e politica, tra le casate. Non ebbe invece tempo, alcuni giorni prima, di recitare il suo epitalamio Ludovico Carbonio a causa delle lunghe trattative in cui gli ambasciatori e rappresentanti delle due casate s'intrattennero per discutere sulla dote della futura duchessa. Esemplare è anche l'orazione pronunciata da Imeneo per le nozze Del Balzo-D’Avalos del 1477, incastonata questa

\footnotetext{
${ }^{50}$ Cfr. Parenti 1976; Dionisotti, 197 n. 2; Pèrcopo. Si veda anche Cruciani 1983, 415: è ivi edito (pp. 421-434) anche il poemetto sul teatro del Campidoglio scritto dal Notturno con il titolo Triumphi de gli mirandi spettaculi et ricche vivande dil solenne convivio fatto da sacri romani al magnifico Iuliano et invicto Laurentio de Medici con il resto creato il sommo pontefice Leon Decimo, con tutta la genealogia et Gloria de Firenza e Roma: composti per Nocturno Neapolitano.

${ }^{51}$ Falletti, "Le feste per Eleonora d'Aragona da Napoli a Ferrara (1473)."
} 
volta all'interno di una struttura drammaturgica e un programma scenografico e coreografico complesso, fatto di carri, monti, torri e personificazioni mitologiche, che — stando al Crispo - era stato elaborato 'faticosamente' da Iacopo Sannazaro per questo evento. ${ }^{52}$ Sannazaro fu probabilmente anche l'autore dell'orazione nuziale, che celebra le virtù del matrimonio e, lodando la nuova 'signora,' onora l'avvenuta unione tra le due casate. Si trattava di un evento politicamente strategico, poiché univa nel sacro vincolo del matrimonio una delle famiglie più fedeli ai re d'Aragona, i D'Avalos, con una delle famiglie più ricche e potenti del regno, i Del Balzo, che non sempre aveva mostrato 'sincera' fedeltà alla monarchia.

La recita oratoria e gli altri omaggi poetici e scenografici coordinati dal Sannazaro, nell'inaugurare il 'mito' di Costanza nella storia, celebrano la corte festante, assimilando entro la recita oratoria i codici della poesia encomiastica e della nuova storiografia umanistica:

Tria maxime trahunt: Pulchritudo et Oculi magnae Constantiae.

Tria maxime cogunt: Amor, Honor et Os magnae Constantiae.

Tria maxime ligant: Benevolentia, Gratitudo et Manus magnae

Constantiae.

Tria sunt suavissima in terris: Musica, Laus et Eloquentia magnae Costantia.

Tria sunt luminaria magna: Sol, Luna et magna Constantia. Illa lucent oculis, dat haec lumen ingeniis.

Tria sunt luminaria magna: Sol, Luna et magna Constantia. Producunt illa corpora, parit haec optimos mores.

Trad. Volpicella:

Tre cose in singolar modo a sé traggono: la Bellezza e gli Occhi della grande Costanza.

Tre cose in singolar modo legano: la Benevolenza, la Gratitudine e la Mano della grande Costanza.

Tre cose sono in terra soavissime: la Musica, la Lode e l'Eloquenza della grande Costanza.

\footnotetext{
${ }^{52}$ La dettagliata narrazione poetica in latino del Moncada è tradita dal miscellaneo Ms. X B 67 (cc. 395-419v) della Biblioteca Nazionale di Napoli contenente la vita di Costanza D’Avalos: Ioannis Thomae Montecatini Adernionis Comitis De Vita Illustris Constantiae Davalos Comitissae Acerrarum. Cfr. Addesso 2012, 82-91. Per la biografia si veda Crispo.
} 
Tre sono i grandi luminari: il Sole, la Luna e la grande Costanza. Quelli splendono agli occhi. Dà questa lume agli ingegni.

Tre sono i grandi luminari: il Sole, la Luna e la grande Costanza. Quelli producono le cose corporee, genera questa ottimi costumi. ${ }^{53}$

\section{Storia. Il poeta cerimoniere: poesia celebrativa e propaganda politica}

[...] con simili inventioni [Sannazaro] divenne altresì familiare al Re, padre del suo Signore, \& ad Alfonso Duca di Calabria, che doveva succedere al Regno. ${ }^{54}$

La partecipazione del Sannazaro al progetto spettacolare del 1477 aveva formalizzato — stando alla parole del Crispo — l'ingresso del poeta fra i familiares del Re, inaugurando, possiamo dire, la prolifica stagione del poeta napoletano anche come cerimoniere della monarchia aragonese e della sua politica festiva. A questo esordio fanno infatti seguito, come abbiamo visto, una serie di eventi di spettacolo che mostrano il Sannazaro impegnato come organizzatore e cerimoniere di feste, come scrittore e persino come attore, delineando il profilo di un poeta versatile esperto dei linguaggi della scena teatrale aragonese ma anche della propaganda politica che sin dal Panormita e dal Facio legava la promozione e lo studio dei classici al senso della magnificenza e della liberalità del governo aragonese e dei suoi sovrani.

Nelle collaborazioni del Sannazaro ai programmi letterario-recitativo-drammaturgico dello spettacolo di corte, il poeta partenopeo riprende alcuni dei temi cari alla storiografia umanistica allora in auge, che promuoveva nuovi valori del vivere sociale e congiuntamente elaborava una nuova immagine del sovrano ideale e delle sue virtù politiche e personali. Essa muoveva dalla necessità primaria di legittimare il governo di una dinastia straniera, com'era nei fatti quella aragonese, e mirava essenzialmente a uno scopo propagandistico volto a vincere le riserve degli intellettuali umanisti locali e delle altri corti italiane che sottolinearono sovente l'indegnità dei re aragonesi sin dal tempo del Magnanimo. ${ }^{55}$

\footnotetext{
${ }^{53}$ Si trascrive da Addesso 2012, 90-91.

${ }^{54}$ Crispo, 11-12.

55 È nota la lettera datata 1 ottobre 1422 di Guarino Veronese (Guarino, 427). Il Flamini documenta anche delle perplessità espresse da alcuna poesia popolare fiorentina sui sovrani
} 
Un furto, ossia quello nel 1447 del manoscritto dei Gesta Ferdinandi regis Aragonum di Valla dalla biblioteca del Re, ad opera del Facio con la complicità del Panormita, è episodio noto che ha dell'aneddotico, ma che rivela in realtà il segno di un cambiamento decisivo e inaugurava l'inizio di un nuovo genere storiografico che risulterà vincente nell'equilibrio tra scrittura della storia e celebrazione encomiastica, o tra narrazione (e rappresentazione) di eventi storici (e spettacolari) e propaganda politica, propendendo per una storiografia glorificante a discapito, se necessario, dell'amore per la veritas perseguita invece da Valla. ${ }^{56}$ Come notava Ferraù nella sua ricomposizione del complesso itinerario degli storiografi aragonesi, "è proprio la storiografia a fornire quella sponda di esemplarità necessaria ad un discorso 'retorico' qual è quello proposto dalla cultura umanistica, una esemplarità, qualche volta espressamente prospettata come tale, qualche volta inserita entro le coordinate della tensione verso un progetto politico ben specifico." 57

La disputa tra il Panormita e il Valla si era già giocata a 'suon di poesia' pochi anni prima sulla scena cortigiana durante i festeggiamenti per il trionfo del Magnanimo nel 1443, di cui entrambi gli storici-poeti scrissero dettagliati resoconti. Per l'occasione, Valla aveva composto i versi per ciascuna virtù rappresentata - la Iustitia, la Liberalitas, la Temperanza — che il Panormita commentò in modo sarcastico, contrapponendovi i suoi componimenti, da lui considerati più degni per il suo Re.

La nuova linea storiografica — basata, secondo l'esempio dei Memorabilia di Senofonte sul modello degli specula principum — esaltava la dignità regia eliminando tutto ciò che sarebbe potuto risultare sconveniente, e presentando invece i sovrani aragonesi come l'incarnazione di ogni virtù attraverso un lungo

\footnotetext{
aragonesi, cfr. Flamini 1977, 131-132. Si veda su questi temi Delle Donne, 257; Ferraù, 43 e sgg.

${ }^{56}$ Come osserva Delle Donne, Alfonso il Magnanimo "è tra i primi — se non il primo in assoluto - signori che, in territorio italico, si dota di storiografi stipendiati apposta per scrivere e raccontare le sue imprese, secondo una prassi che, con ogni evidenza, sembra essere stata importata dalla Catalogna e dalla Castiglia (terra d'origine della dinastia di Trastàmara)." Delle Donne, 255. Lo studioso attribuisce dunque al Magnanimo il ruolo di promotore di una tradizione storiografico-celebrativa che ispirandosi al modello 'ispanico' si diffonde nel regno aragonese e da lì in tutta la penisola. Oltre al Facio e al Panormita merita menzione anche l'opera di Gaspare Pellegrino, medico di Alfonso: cfr. Pellegrino.

${ }^{57}$ Ferraù, X-XI. Si veda anche De Filippis e Nuovo. La validità della storia regia napoletana come modello per altri centri umanistici era già stata sottolineata da Ianziti.
} 
catalogo delle virtutes personali e politiche: la fortitudo, la clementia, la iustitia, la severitas. Questo catalogo fu poi rinnovato dal Pontano nei suoi trattati morali dove, parlando di verità come giudizio morale (e non conoscitivo), insiste sui valori della liberalità, della clemenza e della maiestas, proponendo anche un altro catalogo - quello dei vizi (ad esempio nel De Principe) che il sovrano dovrebbe evitare - che egli integra con la sperimentazione di nuove pratiche di comportamento sociale, in cui si andava definendo anche il ruolo del moderno poeta.

La retorica umanistica sulle virtutes politiche del principe trovava nello spettacolo di corte una sede ideale alle elaborazioni di temi e motivi allegorici e mitologici volti a celebrare, entro il complesso programma spettacolare del cerimoniale pubblico e privato, le reali o presunte qualità individuali dei re e dei duchi e delle rispettive consorti nella costruzione della loro memoria, traducendo nel repertorio figurativo e letterario della drammaturgia cortigiana il nuovo catalogo di virtù del ceto dirigente attraverso la miticizzazione della classicità.

I dati di spettacolo aragonesi in nostro possesso mostrano la farsa nella sua forma encomiastico-allegorica come uno dei generi che meglio si prestava all'esigenze dello spettacolo di corte, riadattando entro una struttura drammaturgica (talvolta semplice, talvolta più complessa) le componenti figurative e recitativo-letterario che caratterizzavano le entrate trionfali sul modello letterario petrarchesco anche grazie all'intervento dell'expertise degli artisti fiorentini. Lo ricorda ad esempio il Notar Giacomo nella cronaca dei festeggiamenti delle nozze tra Beatrice d'Aragona e Mattia Corvino, re d'Ungheria, del 1476, in cui "la fiorentina nacione fe li secte triumphi del petrarcha et girandole." ${ }^{8}$ A questo evento potrebbe legarsi, come ipotizza Addesso, la prima farsa, acefala e adespota, contenuta nel cod. monacense (It. 265) e recitata certamente di fronte a Beatrice d'Aragona, qui lodata per la sua bellezza e per le sue virtù morali, attraverso le personificazioni di Pulchritudo, dell'Honestà ed infine con l'apparizione di Apollo, che a turno con le altre figure recitanti declama i suoi versi e intona il suo canto encomiastico. ${ }^{59}$

La rappresentazione di testi drammaturgicamente strutturati come farse era, come abbiamo visto sin in apertura, pratica ricorrente nei luoghi del divertimento pubblico e privato aragonese. La farsa aveva assunto nello spettacolo di corte

\footnotetext{
${ }^{58}$ Cronica di Napoli del notar Giacomo. Gli artisti fiorentini furono chiamati a Napoli anche per l'allestimento del trionfo alfonsino del 1443, cfr. Iacono, 14.

${ }^{59}$ Il testo è edito da Pieri 1985, 70-74.
} 
un ruolo di rilievo anche forse per la forma duttile e breve, rispetto ad esempio alla commedia di matrice classica, entro cui il poeta cerimoniere di corte poteva elaborare un complesso allegorismo e tradurre in linguaggio teatrale i valori civici, morali e politici elaborati dal ceto dirigente.

Simili alla farsa monacense nella struttura alternante di singoli monologhi recitativi, ma più complesse, sul piano drammaturgico, furono ad esempio le due farse del Sannazaro del 1492, La presa di Granata e Il Triunfo de la Fama, fatte rappresentare a Castel Capuano per celebrare la vittoria in Spagna dei cristiani. La seconda in particolare, Il Triunfo de la Fama, sponsorizzata dal re Federico, fu alquanto ingegnosa e aveva portato dentro la sala di corte carri allegorici con sopra nuovamente figure mitologiche e allegoriche, e l'artificio di un vero arco trionfale, coperto tutto il tempo alla vista del pubblico da un panno nero con gli emblemi dei Re Cattolici e dalla credenza allestita con drappi e argenteria:

[...] era un grande arco triunfale, fatto con colonne e con sculture all'antiqua, con una inscrizzione che contineva le laude delli Ser.mi S.ri Re e Regina de Castiglia, e che per eterna memoria de sì gloriosa vittoria la rep[ubblica] cristiana, vendicata dalle offese de infideli, li dedicava e consecrava quello arco con le spoglie de inimici. ${ }^{60}$

È lo stesso Sannazaro a lasciare una dettagliata descrizione di questa farsa, nella lettera inviata insieme al testo teatrale alla principessa di Altamura Isabella Del Balzo, moglie di Federico d'Aragona, che non potette assistere alle celebrazioni festive. ${ }^{61}$ La minuziosa narrazione conferma l'attenzione del Sannazaro al linguaggio teatrale nella duplice dimensione visuale e verbale della messinscena. Da 'sotto' l'arco esce dunque la dea Pallade (o Minerva, come pure "m'appellan"), riccamente vestita con indosso i simboli e gli emblemi identificatori secondo l'immaginario classico, che Sannazaro spiega accuratamente nella forma e nel significato alla principessa Isabella. Con voce audace e sonora, la dea si rivolge dunque ai due sovrani, il Re e la Regina, e nel presentarsi elogia la corte festante — "i suoi seguaci” — per la Prudenzia, la potenza e il valore che ella ha acceso in loro, stimolando la loro

\footnotetext{
${ }^{60}$ Qui e di seguito si cita dall'edizione Mauro, 286-295.

${ }^{61}$ La missiva è conservata in alcune trascrizioni posteriori: Monaco, Staatsbibliothek, It. 265; Napoli, Biblioteca Nazionale, X, C, 45; XVI, D, 14; Branc. III, a, 9; Roma, Biblioteca Apostolica Vaticana, capp. 73. Cfr. Nocilli, 168.
} 
audacia e prontezza nelle armi, per il raggiungimento della Fama e della gloria in tutte le terre che sono ora liberate dall'assedio di Maometto:

Ferrante il puse in bando di Siviglia

Ferrando di Castiglia or l'ha cacciato;

Ferrando è ancora serbato a far vendetta

della gente maldetta; e questo nome,

non dico quando e come, avrà corona

in Grecia, in la Velona, in la gran sede

c'ogge il Turco possede; e'n la Soria,

in India, in Barbaria, in ogni parte

ove Maumetto ha sparte le sue spine.

Felice e peregrine anime degne!

Felice quelle insegne e quella guerra!

Felice il ciel, la terra, e 'l giorno e l'anno

Che tanto ben vedranno! O spirti grandi,

o Alfonsi, o Ferrandi, o Federichi,

pensate a' vostri antichi, e per quell'orme

sequite le mie norme. Ecco qui 'l pregio,

ecco qui l'alto segio, il qual vi chiama,

ecco qui la gran Fama trïunfante

che vien con sue volante argute penne,

lodando il re che sì gran palma ottenne!

40

Introdotta dai versi di Pallade e trainata da due giganti "molto alti, armati al modo antiquo," entra dunque trionfalmente il carro della Fama, personificata da una figura "in la forma che stanno le statue," con indosso una corona di alloro e amaranto sul capo, e con grandi ali d'oro in cui erano dipinti - descrive sempre il Sannazaro con perizia nei dettagli - tanti occhi, orecchie e lingue. ${ }^{62}$

${ }^{62}$ Dei versi recitati dalla Fama si componeva, come abbiamo visto, anche la Rappresentazione allegorica mantovana del 1495 di Serafino Aquilano, rappresentata di fronte al Duca di Calabria, Ferrandino e al Marchese Gonzaga. "[...] decentissimamente ornata, cum l'ale e due trombe in mano, cum li pennoni araginesi e gonzagheschi," l'iconografia della Fama presenta generiche affinità con la Fama della farsa del Sannazaro, che meritano tuttavia di essere maggiormente investigate. Tuttavia lo schema dell'alternanza dei monologhi in endecasillabi con rimalmezzo mostra una significativa affinità della rappresentazione mantovana con il modello aragonese che merita di essere ulteriormente investigata. Inoltre Serafino sembra riproporre nella nuova sede 
Con voce alta si presenta al suo pubblico regale, e nel celebrare il potere della sua voce - che "tanto si spande, / e nel parlar tanto più forza acquisto" (vv. 84-85) — la Fama inizia il suo tripudio per l'aragonese stirpe, per dare "immortal memoria" dell'impresa ("alta e onesta") e della vittoria dei principi e di tutte le loro virtù e le loro grazie (vv. 129-130).

Però ciascun meco lode e ringrazie

il ciel che ne mostrò tant'ardimento,

che di parlarne mai non seran sazie

queste mee cento bocche e lingue cento.

L'eterna gloria e memoria dei principi d'Aragona è poi 'custodita' dal canto di Apollo che, entra "legiadramente vestito," con indosso "una bella mascara, coi capelli biondi e lunghi che li pendeano dietro le spalle" e con una corona di alloro nella testa. Il potere memoriale del canto è celebrato da Apollo stesso, che, tuttavia, ammonisce ed esorta poeti e storici a saper usare uno stile chiaro, dal "parlar gentile" se essi vogliono con i loro versi rendere immortali le regali imprese:

[...] Ogne gran cosa,

quantunca glorïosa et eccellente,

quantunca fra la gente sia pregiata,

si non è illustrata in chiaro stile

d'un bel parlar gentile in la mia corte,

subito sente morte. Inde fo lieti

istorici e poeti, e loro affanni

vivon mille e mill'anni. Oh benedette

anime in celo elette, e nate tale

de fare altre immortale e sé beate!

Ché poi che questa etate è frussa e breve,

sforzar ciascun si deve con soi gesti

in far c'al mondo resti il nome vivo

per quel che 'n carta io scrivo; onde quel grande,

c'ora il suo nome spande in tanto onore,

cortigiana lo schema della farsa aragonese con la medesima funzione politica e encomiastica che nell'occasione festiva sigillava l'accordo tra i due signori di Mantova a e Napoli. Cfr. Addesso 2013, 10-22. 
che 'l barbaro furore ha superato

e 'l suo regno espurgato dalla iniuria

de la moresca furia, avrà gran parte

di fama in le mie carte, e per istoria

vedrà la sua vittoria celebrare.

Le parole di Apollo iniziano dunque con una riflessione sulla Poesia e Storiografia, sulle possibilità del Canto e degli scritti, e sul ruolo del moderno Poeta e Storico, esortando il poeta a sforzarsi con il suo gentil canto e "coi soi gesti," ossia con la sua presenza di corpo e voce, di rendere memorabili i suoi scritti a celebrazione ("per istoria”) della fama della odierna vittoria aragonese sui mori. Ancora Apollo, rivolgendosi direttamente al suo pubblico - nella forma allocutoria comune, come abbiamo visto, a molte farse — incita la regina all'ascolto, emotivamente partecipe del canto e l'invita a guardare ("or mira, mira, et in qua gli occhi gira") il trionfo del suo "caro sposo" e con lui del duca Alfonso e del Re Ferrante.

\section{[...] E poi cantando}

dirò 'l novo Ferrando, il cui valore

con eterno splendore e degni esempi

farrà leti i soi tempi, e di trofei

ornarà gli alti Dei $[\ldots]$.

La farsa si conclude, dunque, con il canto vero e proprio di Apollo accompagnato da una viola da braccio; segue la danza di un matto al ritmo di un tamburino; quindi l'entrata al suono di pifferi e trombetti di Federico d'Aragona insieme con quattro danzatori-mimi vestiti con il cimiero e i colori (nero e paonazzo) della casa aragonese; l'omaggio coreutico e in versi castigliani di una donna vestita a la francese' di verde e infine con i fuochi pirotecnici a chiusura della festa.

Lo spettacolo fu grandioso a giudicare anche dalle cedole di tesoreria. ${ }^{63}$ Presentava una raffinata struttura drammaturgica elaborata dal Sannazaro con grande ricchezza e puntiglio nei particolari: per l'artificio degli apparati scenografici e mobili; per l'inclusione di parti coreografiche, mimiche e musicali; per i costumi, le insegne, le maschere; per la componente verbale che, nella sequenza dei monologhi recitativi delle figure mitologiche e allegoriche, aveva affidato ad Apollo e al suo canto all'antica il messaggio politico a celebrazione delle virtù

${ }^{63}$ Nocilli, 171 e Appendice. 
del principe ad eterna memoria dell'impresa dei sovrani d'Aragona. La storia era dopotutto, secondo quanto scritto dal Pontano in ripresa degli antichi, quasi una poesia in prosa, che poteva presentarsi oltre che nella forma di opera propriamente storiografica anche in quella di narrazione storica (o mitologica), di poema o ancora, spostando lo sguardo sulla scena aragonese dello spettacolo di corte, anche nella forma della farsa.

\section{Poesia. Il poeta umanista: l'Orfeo napoletano}

Interprete dell'Apollo sannazariano del 1492 e del suo canto accompagnato alla viola potrebbe essere stato il celebre cantore alla lira Serafino Aquilano (14661550), in quegli stessi anni a Napoli chiamato da Fernandino fra gli accademici pontaniani, o il barcellonese Cariteo (1450-1514), che, come abbiamo visto, col Sannazaro condivise la scena teatrale cortigiana sia come attore di farse che come 'ordinatore' delle messinscena delle sue 'frottole' e di altra poesia recitativa.

Erano giunti nel Regno a circa dieci anni di distanza: l'uno, il Cariteo, fra il 1467 e il 1468 all'età di diciasette-diciotto anni, ricoprendo incarichi amministrativi sin dai primi anni Ottanta, come risulta anche dal suo Canzoniere (sonetto XCI); ${ }^{64}$ l'altro, Serafino, nel 1478, condotto dallo zio presso la corte di Potenza di Antonio de Guevara dove studiò musica sotto la guida di Guglielmo Garnier. ${ }^{65}$ I nomi dei due poeti-cantori s'intrecciano ripetutamente nei ricordi degli umanisti e, fra tutti, nelle pagine del Calmeta, che li porta in più occasioni come esempi di perfezione della poesia recitativa contemporanea. Il 'critico' fa inoltre risalire al Cariteo la moda cortigiana degli strambotti, diffusasi per tutta l'Italia grazie anche allo straordinario successo dell'Aquilano, che si cimentò con il genere dopo aver sentito a Milano, verso il 1490, un altro musico napoletano, Andrea Costa, intonare accompagnandosi con la lira i versi del poeta barcellonese.

i quali [Cariteo e Serafino] sonosi sforzati d'accompagnar le rime con musica stesa e piana, acciocché meglio la eccellenza delle sentenziose e argute parole si potesse intendere, avendo quel giudicio che suole

\footnotetext{
${ }^{64}$ Cfr. l'Introduzione di Pèrcopo all'edizione da lui curata di Gareth; cfr. anche Parenti 1993; Barbiellini Amidei, 3; Fenzi 2002, 117-140.

${ }^{65}$ Costretto a rientrare all'Aquila a seguito della morte del padre, Serafino è di nuovo a Napoli tra il 1485 e il 1489 e poi di nuovo tra il 1491 e il 1492. Su Serafino si vedano i preziosi studi Rossi; Ciminelli (Aquilano) 2005; Ciminelli (Aquilano) 2002; Dell'Aquila e Bauer-Formiconi.
} 
avere un accorto gioielliero, il quale, avendo a mostrare una finissima e candida perla, non in drappo d'oro la tenerà involta, ma in qualche nero zendado, a ciò che meglio possa comparire. ${ }^{66}$

Qui, probabilmente, Calmeta tenta di risolvere l'ambiguità di fondo della cultura umanistica tra poesia orale e modello alto e scritto dell'esercizio lirico che, se privilegiava il testo poetico rispetto all'esecuzione musicale, legava ancora la poesia all'atto performativo del cantore, considerando la forza espressiva della poesia orale. Alla poesia è così ancora riconosciuto il carattere primariamente di esecuzione vocale alla quale il testo poetico si affidava unendosi al canto accompagnato alla lira, su cui la cultura umanistica proiettava i valori tratti dall'antichità, nobilitando la pratica diffusa di improvvisatori e cantori attraverso un processo di personificazione con l'archetipo che riattivava in poesia e sulla scena teatrale cortigiana l'immagine di Apollo o del mitico cantore Orfeo, simbolo della poesia civilizzatrice.

Celebrati dalla cultura umanistica insieme ad altri poeti-cantori dell'epoca come i nuovi Orfei, Serafino e Cariteo furono essi stessi artefici del loro mito che elaborarono attraverso la loro arte poetica e scenica. Serafino stabilisce il legame con l'antico sulla scena teatrale, attraverso le sue performance nei luoghi strategici dell'intrattenimento elitario di corte. Egli attinge dai materiali della tradizione, usa la tecnica del travestimento e avvia un processo di tipizzazione del proprio alter ego artistico che prende vita dalle proprie originali caratteristiche espressive e competenze attorali. Muovendosi sull'uso del volgare dei modelli lirici più diffusi, accompagnando il suo canto alla lira in base a schemi melodici noti, Serafino troverà un momento privilegiato per l'elaborazione della sua maschera di cantore nel cimento del genere pastorale che, attraverso l'allegorismo bucolico e il richiamo al mitico cantore tracio, gli consentì di amplificare la sua identità attorica nell'immagine degli antichi cantori e di affidare al canto il suo messaggio politico. ${ }^{67}$ Anche il Cariteo nobilita la sua arte di performer nel richiamo all'antico Orfeo e al suo immaginario mitico. ${ }^{68}$ Egli opera in maniera selettiva e programmatica all'interno

\footnotetext{
${ }^{66}$ Calmeta, 22.

${ }^{67}$ Sui processi creativi che l'Aquilano mette in atto nella scrittura pastorale e sulla scena a corte per costruire il suo personaggio, la sua maschera di cantor, e il suo canto (in questo caso di attacco) politico, mi sono occupata nel saggio "Serafino Aquilano and the Mask of Poetd: A Denunciation in the Eclogue of Tyrinto e Menandro (1490)," in corso di stampa.

${ }^{68}$ Il Cariteo fu anche compositore, almeno in un caso, della parte musicale. Abbiamo infatti un ms. e un incunabolo in cui, per lo strambotto Amando e desiando io vivo e sento, il Cariteo
} 
delle sue rime, destinando l'evocazione della voce orfica e della sua lira principalmente alle canzoni 'civili' poste in Appendice alla raccolta poetica intitolata l'Endimione, edita nel 1506 e poi in una nuova versione nel $1509 .{ }^{69}$

Le due canzoni 'morali' VI e VII, rispettivamente Aragonia e La candida vertute al cielo uguale, furono scritte tra il 1495 e il 1496 in elogio dei principi aragonesi per le loro virtù nella guerra, per la loro generosità e magnanimità, per la fede, la costanza e la liberalità. Cariteo accantona, dunque in questa fase, la poesia amorosa per dedicarsi a quella civilizzatrice e morale. I suoi versi esaltano il dono della parola e dell'eloquenza, e affidano al suo canto e a quello degli amici poeti e dotti letterati — sul modello fiorentino ficiniano (in ripresa della IV egloga virgiliana) — il compito di far rinascere una nuova età dell'oro. Il poeta riconosce alla poesia una funzione primariamente pedagogica e civica nella società moderna e la elabora attraverso significati plurimi per mezzo dell'allegoria e dell'immaginario mitologico. ${ }^{70}$

Nella VI canzone, ad esempio, il Cariteo annuncia in una profezia che Ferrandino rinnoverà l'età aurea riportando in gloria il regno come fece Augusto:

Per te dée rinovare un secol d'auro,

Qual per campi e città del regio Latio

In tempo di Saturno andar solea.

E nella canzone VII sempre a Ferrandino, poeta anch'egli fra i poeti, il Cariteo consegna la lira e, in celebrazione delle sue virtù, assimila il suo canto a quello del mitico cantore tracio:

Orpheo che col soave et alto tono

Di tua voce, et col sono

D'esta lyra immortal, movendo i passi,

Si trahea presso i boschi, i monti e i sassi

Come l'Apollo sannazariano del 1492, Cariteo affida alla poesia orale, e al suono del suo canto e del suo incedere ritmico, il compito della memorabilità delle

è indicato anche come autore della musica. Vedi Cattin, 273-290, 305-313, 285. Cfr. Anche Gerbino 2012, 315-343.

${ }^{69}$ Cfr. Caracciolo Aricò.

${ }^{70}$ Ventrone 1992. 
grandi imprese dei principi aragonesi. Attraverso il suo canto di elogio il poeta mira a rendere eterna la sua fama. Il nome del Cariteo è destinato cioè a 'rifulgere' proprio perché i suoi versi ricordano lo splendore dei suoi re e similmente dei loro sommi poeti. Fra tutti il nome del 'suo' Sannazaro ritorna più volte nei versi del poeta barcellonese: "Là conven che 'l mio nome splenda e luca' [...] rimembrando l'onor ch'al cielo extolle / il mio bel Sannazar" (Pascha I, 40). ${ }^{71}$

La poesia conferisce la fama rendendo il poeta immortale. Essa ha la virtù di imporre la propria intrinseca grandezza alle cose, come teorizzava in questi stessi anni il Pontano nei suoi Dialoghi e fra tutti nell'Actius, incentrato sulla riflessione intorno alla Poesia passando attraverso la discussione delle attività 'sorelle': l'oratoria e la storiografia.

Ac tametsi oratoris quoque est aliquando et magnifice et excellenter, tamen id non ubique, neque semper, cum poetae hoc ipsum ubique suum sit ac peculiari, etiam cum in minutissimis atque humilibis versatur rebus.

Trad. Ed ecco, anche quando un oratore è magnifico e di eccellenza, tuttavia questo non avviene sempre e in ogni situazione, il poeta è sempre straordinario anche quando tratta di cose minori e umili.

Il poeta è innanzitutto generatore di grandezza; la sua arte poetica non è mediatrice, moderatrice o imitatrice della realtà naturale, bensì emulatrice e "nell'emulazione scopre - come commenta Tateo nella sua lettura delle poetica pontaniana — la sua superiore qualità, la sua essenza spirituale ed umana, che si riflette nell'effetto di gran lunga più alto cui può giungere." ${ }^{72}$ Le metafore e le favole danno la miglior misura dell'arte del poeta e della sua capacità di creare nuovi universi, lasciando alla Poesia l'unico compito di esprimere la grandezza del Poeta.

Questa immagine del nuovo poeta umanista è riconoscibile ancora nei versi del Cariteo e in particolare nella canzone VIII, Già se dissolve homai la bianca neve, dove il poeta manifesta il suo desiderio di ammirazione e gloria eterna che egli può

\footnotetext{
${ }^{71}$ Il Libro intitulato Pascha è un poemetto in terzine scritto dopo il 1503 (poiché allude al Pontano come già morto), in cui è stata riconosciuta, al pari di altri componimenti scritti in questo periodo dal Carieto, una impostazione più chiaramente neoplatonica. Cfr. Barbiellini Amidei, 53.

${ }^{72}$ Tateo, 119.
} 
forse raggiungere, al pari del Sannazaro, invocando Apollo e Orfeo per elevare il suo stile nel canto in lode della gloria degli Aragonesi e del suo signore Ferrandino:

[...] Che fusse d'amor libera l'alma,

Forse ch'io spererei dal ciel tal dono,

Qual diede il sacro Apollo al Thracio Orpheo;

Ond'io direi con grave, eroico suono

Gli altri Trophei, la gloriosissima palma

Di quel ch'è 'n terra più che semideo.

Forse di Caritheo

Vivrebbe il nome allhor non men preclaro,

che quel del Sannazaro. ${ }^{73}$

Come osserva Caracciolo Aricò, "nel doppio segno di Apollo e di Orfeo si ripete l'investitura che unisce inscindibilmente, nella visione di Benedetto Gareth, la dinastia e i dotti," identificando le sorti dell'uno e dell'altro in un unico destino.

Il Cariteo, poeta acclamato e funzionario di spicco della monarchia d'Aragona, s'identifica egli stesso con il cantore mitico Orfeo ed evoca l'immagine di Apollo a celebrazione del suo canto poetico: un canto promotore d'impegno civile poiché dispensa la gloria (politica) e garantisce la fama eterna al principe, rendendo per l'appunto eterno il suo nome, che può così essere annoverato nel nuovo Parnaso aragonese e ricordato dal canto e dall'inchiostro dei "famosi poeti."

Viva il mio nome in bocca di famosi poete, ch'io non men che divi adoro, e voi narrate i van sogni vinosi.

Parle di me il Pontan, quel bel thesoro d'Apollo e de le Aönide sorelle che con la lingua sparge un fiume d'oro.

Depinto io sia ne l'opre eterne e belle

del mio bel Sannazar, vero Syncero, ch'allhora io giungerò fin a le stelle.

E 'l lume d'Aristotile d'Homero

mi laude, io dico Pardo insigne e chiaro,

\footnotetext{
${ }^{73}$ Oltre al citato studio di Caracciolo Aricò si veda Fenzi 2005.
} 
per gemino idïoma al mondo altero.

Altilio e Galateo, physico raro,

e Summontio d'ingegno e di vertute

ornato, et a gli amici dolce e caro.

E Musephilo e Maio, anime argute,

ciascun Quintilïano al secol nostro,

moderator de l'aspra gioventute.

E tu, Corvino mio, poi ch'io ti mostro

che di sangue e d'amor son teco giunto,

parla di me con penna e con inchiostro. ${ }^{74}$

Il Gareth celebra in poesia il suo stesso mito e lo lega a quello di una casta eletta e distinta di poeti moderni in aperto dialogo con l'antico. Come è noto, il suo nome ricorreva, attraverso un gioco di citazioni e rimandi caro agli umanisti non solo di casa aragonese, negli scritti degli amici e colleghi che egli frequentava fra l'accademia pontaniana e le sale dello svago e dell'amministrazione politica aragonese. A lui il Pontano dedica il De splendore, e un componimento degli Hendecasyllabi seu Baiae, in cui ricorda l'origine classica del suo nome derivante dalle Charites, ossia dalle Grazie (Ad Chariteum, I, XXX). Di lui lascia anche un ricordo nel De sermone, riferendo dell'arguzia dei suoi versi ed apprezzando il suo umorismo e il suo 'ingegno.' Egli è inoltre presente nei dialoghi pontaniani, l'Aegedieus, l'Antonius, e ancora nell'Asinus. Mentre il Summonte ricordava in una lettera ad Angelo Colocci del 28 luglio 1515 come "lo amico [Cariteo] si dilettava a parlare poeticamente." ${ }^{75}$ E proprio a Summonte (lo stampatore), il Sannazaro affiancava il Cariteo (il poeta e improvvisatore) nella sua Arcadia (egloga XII), facendo loro indossare la maschera pastorale rispettivamente dei pastori Summontio e Barcinio per ricordare, rinnovare, diffondere e celebrare ad eterna memoria il canto e gli

\footnotetext{
${ }^{74}$ Si trascrive da Fenzi 2005.

${ }^{75}$ Cfr. Gareth (Cariteo), XLV-XLIX e, in Appendice al vol. I, CCXCIII-CCXCVI. Cfr. anche Barbiellini Amidei, dove la studiosa riporta anche i ricordi sul Cariteo come uomo erudito e dotto fra gli accademici pontaniani, rispettivamente di Egidio da Viterbo nella sua Historia viginta saeculorum, e del Galateo in una lettera a Belisario Acquaviva (11-12 e nota). Sulle testimonianze del Calmeta, si veda la prosa Qual stile tra' volgari poeti sia da imitare, e la Vita di Serafino Aquilano, trascritte nell'ed. Grayson, rispettivamente alle pp. 20-25 e pp. 60-77. Ma ancora altri ricordi possono rintracciarsi nelle scritture di fine secolo.
} 
accenti uditi dal sommo Pontano alias Meliseo, attraverso le loro rispettive arti di stampatore e improvvisatore.

Il nome del Pontano, unitamente a quello del Sannazaro e di altri pochi eletti poeti e sapienti della selva Antiniana (la nota villa del Pontano), ricorre con programmatica presenza lungo tutta la raccolta del Cariteo intento a intonare di volta in volta i canti di gloria degli Alfonsi e dei Ferranti alla presenza di Apollo e delle sue Muse, come 'sintetizzato' nella canzone XVI:

Hor è nel suo soggiorno

Apollo con le nove alme sorelle:

Hor quella insigne, sacra, alta dottrina,

chiamata humanità, sola divina,

Ferirà con la testa l'auree stelle;

Le selve Antiniane in varii canti

Risonaran la gloria degli Alfonsi

Et d'inclytu Ferranti [...]

Il travestimento mitologico della selettiva cerchia dei poeti pontaniani era dopotutto episodio significativamente ricorrente della festa aragonese durante la quale la corte si trasfigurava in un nuovo Parnaso nella duplice o alternativa presenza di Apollo e di Orfeo. Lo abbiamo visto con la farsa sannazariana del 1492. Ma altri 'moderni' Orfei, locali e stranieri, si erano alternati con le loro performances poetiche accompagnate ora con il liuto, ora con la lira o con la viola anche durante i festeggiamenti per l'unione nuziale fra Eleonora D'Aragona ed Ercole I d'Este nel 1473: il noto liutista ferrarese Pietrobono, giunto a Napoli con la delegazione d'Este per prendere Eleonora e condurla dal futuro sposo; e il fiorentino Aurelio Brandolini, l'“Orpheus Christianus” che, a Napoli dal 1466, aveva compiuto i suoi studi e raggiunto la sua fama di poeta e cantore, secondo quanto narra suo fratello Raffaele in una breve biografia. ${ }^{76} \mathrm{E}$ ancora, Apollo e le Muse scesero dall'Olimpo a onorare la principessa Beatrice d'Aragona nei festeggiamenti del 1476, che videro con buona probabilità — lo ricordiamo — la recita della farsa acefala del codice monacense. Con l'augurio d'ispirazione poetica per la giovane D'Avalos, Apollo si presenta con in dono una cetra d'oro anche alla corte festante di Costanza e di Federico Del Balzo nel 1477. Ed evocando il canto

\footnotetext{
${ }^{76}$ Atlas, 102, 108-109. Su Aurelio Brandolini si veda anche Mayer; Haraszti, 54. Sul fratello minore, Raffaele, si rimanda alle note dello studio di Guarino 2010, 350.
} 
di Orfeo, il Perleoni celebrava "in veste di pastore" il Duca Alfonso di Calabria per la sua vittoria sui turchi a Otranto nel 1482. Ancora di una rappresentazione di Orfeo con la lira "che con grande arteficio suonava" in atto di ammansire le fiere e con una cornucopia in mano dalla quale uscivano monete d'oro e d'argento per il popolo, racconta il Summonte nel ricordo dei festeggiamenti per l'incoronazione di Alfonso II nel 1494..$^{7}$ Mentre un Apollo arriva a corte a predire un radioso futuro al giovane Ferrandino, recitando, questa volta insieme a Giove, i versi "in gran dottrina” a diletto di Isabella Del Balzo durante i festeggiamenti per la sua sosta a Barletta nel 1496 prima di raggiungere il suo sposo Re Federico per la successione al trono del Regno di Napoli. ${ }^{78}$

L'elogio rivolto da Apollo al principe creava una relazione diffusa nella cultura umanistica di matrice ficiniana tra Principe/Apollo/musico che nella performance attivava uno stretto legame tra il mecenate e l'artista e una reciproca legittimazione ed elevazione delle rispettive 'identità' pubbliche, conferendo alla poesia teatrale e al poeta-performer un valore primariamente civile. La presenza del suonatore di liuto o del cantore alla lira nei luoghi dell'intrattenimento proiettava sulla scena performativa delle cerimonie accademiche o dello spettacolo di corte l'immagine antica del mitico cantore, rinnovata nei testi e nelle pratiche degli umanisti, trasfigurando la sala entro una dimensione mitica. Il cliché di Orfeo, di Apollo o di Anfione diviene la "maschera della forza del recitare," del potere memorale della parola detta, cantata, enunciata, che la drammaturgia cortigiana assimila e ripropone in una nuova forma teatrale all'antica alternativa alla commedia classica.

Le figure di Orfeo e Anfione sono invocate in maniera eloquente anche nella farsa allegorico-morale Imagico (o il Magico) di Pier Antonio Caracciolo, ${ }^{80}$ a preludio della disputa filosofica, tra le ombre di Diogene e Aristippo evocate dal mago-negromante con la sua arte magica e condotte da Caronte insieme al giudice Catone al cospetto del Re Ferrante e della sua corte. I due antichi filosofi introducono i rispettivi modelli di vita — l'una lontana dal viver civile, l'altra volta invece verso "altra mensa" per godere delle ricchezze e della dolcezza della vita — e le loro

\footnotetext{
77 Summonte, t. III, 481 e sgg. Si cita da Addesso 2012, 68.

${ }^{78}$ Rogeri De Piacenza di Nardò.

${ }^{79}$ Guarino 1999-2000, 13.

${ }^{80}$ La farsa del Magico è tràdita dal citato manoscritto monacense e fu edita dal Torraca e ora da Cristiana Anna Addesso (Addesso 2012, 110-117).
} 
posizioni si traducono in una riflessione sulla relazione tra gli intellettuali e il potere che Catone media con il suo "bel parlar soave, honesto et saggio," concludendo in omaggio al re che "il viver felice se retrova / sol dove virtù cova."

Dopo un lungo monologo a difesa della "Nigromanzia" come arte basata su un'ampia conoscenza delle diverse discipline e di "doctrine / quasi tutte divine," il personaggio del Magico aveva infatti annunciato l'apparizione degli spiriti dei due filosofi in una sorta di scena rituale in cui, evocando Ecate (lodata dea anche degli Inni orfici), celebra i mitici cantori e il potere del loro canto insieme a quello del profeta Giosuè:

Chè come hebe potentïa nel sole Josuè con le parole finché gloria hebe da soa victoria, et Amphïone

col suo dolce sermone i saxi spense perfin che Thebe cense d'alte mura, Orpheo con la mesura del suo canto et dolceza del pianto per costumi solea fermar i fiumi et le montagne andar per le campagnie et fece actento l'inferno al suo lamento et fe' dormire Cerbaro col suo dire et dè dilecto ov'è tanto dispecto, cossì ancora te prego, senza dimora sia rimoto dal cielo ogne contrario vento, ogn'altra cosa creata si riposa finchè dura questa breve coniura che farragio.

Il Mago-negromante (ma anche astrologo, teologo o filosofo e di tutte le arti e doctrine esperto), s'identifica con i due mitici cantori e l'antico profeta e attraverso questi legittima e nobilita la sua arte magica e insieme le sue parole di poeta e narratore che - come dirà rivolgendosi in forma diretta a Ferrante nel monologo conclusivo della farsa - mirano non tanto a raccontare di grandezza, gloria, vittoria, clemenza o del grande ingegno che oggi nel Regno detengono i sovrani aragonesi, non solo a narrare della fama delle virtù che si 'spande' in ogni parte, bensì, lontane da ogni intento di adulazione, vogliono "dimostrare et dire" ciò che non è conosciuto, annunciando come assoluta verità un futuro in cui la Fortuna 
sarà favorevole, grazie alla sua prudentia, e sarà così privo di guerre, di furia, penuria e invidia.

Entro la struttura drammaturgica della farsa, ormai collaudata sulla scena teatrale aragonese, Caracciolo ordisce una sofisticata pièce mitologica e filosofica, di chiara matrice ficiniana, attraverso la quale il poeta-Imagico glorifica il re Ferrante al cospetto del quale l'autore-attore recita la parte del protagonista, dando al suo dire — sebbene umile nello stile ma alto nella dottrina — un valore eternizzante. $^{81}$

Io non so' adulatore o hom che sognia fabulecte et mensogne, perché esperta è mia doctrina et certa, et però spero che tu tenerai per vero il meo parlare.

Io non voglio narrare a lo presente quanto è alta et potente la toa gloria, né dir de toa victoria et toa clementia, de l'invicta potentia et claro ingegnio che tanti anni il tuo regno hai governato in pacifico stato et in divitio, né men de toa iustitia in ogne gesto. E però s'io me resto recontare le toe virtù preclare e cha se spande per sé toa fama grande in ogne parte, ma voglio dimostrarte et dire cose che te son forse ascose, ché nel volto cognosco zò ch'è occolto et quanto il cielo nasconde col suo velo. [...] Allora ogne acra guerra, ogne aspra furia, allora ogne penuria et ogne invidia, allora ogni perfidia, ogne ramarico che fosse el mondo car(r)ico et oppresso essendo ad lui sommesso cesseranno. Et per non dar più affanno ad tanta alteza

\footnotetext{
${ }^{81}$ Sull'influenza del pensiero ficiniano nella farsa del Magico del Caracciolo e più in generale della sua ricezione nella cerchia pontaniana, si rimanda al recente saggio di Soranzo e alla bibliografia interna.
} 
con l'infirma bassezza del mio dire

Io voglio qui finire e poi basarte

Toa sacra mano prima che me parte.

Il giovane Caracciolo - figlio del noto petrarchista Giovan Francesco celebrato nell' Arcadia sannazariana come 'poeta civile' (seguendo l'interpretazione del Santagata) — , fu autore di numerose farse, oggi perdute, che egli recitò personalmente per il suo pubblico di corte. Lo abbiamo già incontrato nel ruolo dell'araldo nella farsa recitata di fronte al duca Alfonso di Calabria, probabilmente a Castel Capuano, e ancora in quella del messaggero turco, rappresentata dal Caracciolo di fronte questa volta alla principessa Sanseverino di Bisignano. ${ }^{82} \mathrm{E}$ fu forse in questi luoghi, alla corte teatrale dei Sanseverino, che il nostro "poeta comico regio,” divenne familiare di un altro attore, Francesco de’ Nobili, detto Cherea, segretario privato di Ferrante Sanseverino, che nel suo repertorio di scena presenta una farsa intitolata El Mago, identificata dal Padoan proprio con quella del nostro Caracciolo. ${ }^{83}$ A dispetto delle poche notizie biografiche che del Caracciolo ci sono rimaste e della dispersione dei suoi testi dovute a gelosie e competizioni degli eruditi studiosi, che rendono ancora indefinita la figura di questo poeta e performer della corte aragonese, l'arte itinerante e mobile dell'attore garantì la circolazione della sua opera, sradicandola dal contesto teatrale aragonese e proponendola sotto nuova forma e con una rinnovata funzione nelle recite di nuove feste e nel nuovo mercato editoriale lagunare.

È ormai nota l'istanza del Cherea al Senato Veneto nel 1508 di stampare i numerosi testi che egli aveva, come dichiara, "cum spesa, verdù e travaglio raccolte e traducte de greco e latino in vulgare a comodità et satisfatione de qualunque persona [...] tragedie et comedie, Egloge et tragedie." ${ }^{84}$ Cherea non sfrutterà mai il privilegio di stampa, ma certamente grazie al suo repertorio teatrale, costruito

\footnotetext{
${ }^{82}$ I titoli delle altre sue farse sono: Farsa del mercatante che vende due schiavi, uno masculo et una femina; Farsa del malato, la matre e due famigli con un medico e un prattico; Dialogo di due pezzenti; Colloquio di un villano, due cavaiuoli e uno spagnuolo; Un medico, un villano e la mogliere del villano; Quattro villani che acconciano le loro mogli con altri. Farsa di una cita, lo cito, una vecchia, uno notaro, lo preite con lo yacono et uno terzo.

${ }^{83}$ Padoan, 37.

${ }^{84} \mathrm{Il}$ passo successivo sarebbe stato la richiesta di recitare le sue commedie nella loggia del Rialto, luogo nevralgico della città, "espressione di esigenze di rappresentanza dell'oligarchia mercantile, e delle pulsioni del mercato circostante." Guarino 1995, 165.
} 
sulle esperienze cortigiane e presentato nei luoghi dell'intrattenimento di corte, si consolida nello scenario festivo lagunare la presenza del teatro cortigiano e la progressiva assimilazione e codificazione della commedia 'regolare. ${ }^{85}$

All'indomani della caduta della dinastia, la 'diaspora' dei poeti, diplomatici, cerimonieri e intrattenitori degli Aragona, costretti a muoversi verso altri lidi, favorirà la circolazione dei materiali testuali e scenici che nella Napoli quattrocentesca erano nati da una sostanzialità orale entro la cornice politica della festa, privata e pubblica, degli Aragona, portando le esperienze del teatro cortigiano, nelle forme tipiche della farsa, della 'commedia nova' o della poesia pastorale, in nuovi contesti festivi, che producevano risultati diversi nello scenario teatrale peninsulare cinquecentesco e nel nascente mercato editoriale.

University of Leeds

\section{Opere citate}

Addesso, Cristiana Anna. Teatro e festività nella Napoli aragonese. Firenze: Olschki, 2012.

"'Portano i latron pieni i lor saini': elementi della teatralità aragonese nella produzione egloghistica e farsesca di Serafino Aquilano e Antonio Ricco." Rivista di Letteratura Teatrale 6 (2013): 10-22.

Anglés, Hygini. "Alfonso V d'Aragona mecenate della musica ed il suo ménestrel Jean Boisard." Scripta musicologica, vol. II, Roma: 1975. 765-778

Atlas, Allan W. Music at the Aragonese Court of Naples. Cambridge: Cambridge University Press, 1985.

Barbiellini Amidei, Beatrice. Alla Luna. Saggio sulla poesia del Cariteo. Firenze: La Nuova Italia, 1999.

Bauer-Formiconi, Barbara. Die Strambotti des Serafino dall'Aquila: Studien und Texte zur italienischen Spiel- und Scherzdichtung des ausgehenden 15. Jabrbunderts. Munich: Fink, 1967.

\footnotetext{
${ }^{85}$ Leredità di questo articolato componimento allegorico-filosofico del Caracciolo di matrice aragonese sembra protrarsi nei secoli, come indica l'investigazione di Gianni Cicali sul repertorio del commediografo napoletano, Pietro Trinchera, che mostra, secondo lo studioso, ne La Moneca fauza evidenti richiami alla farsa del Magico del Caracciolo, cfr. Cicali, 133-201.
} 
Bentley, Jerry H. Politics and Culture in Renaissance Naples. Princeton: Princeton University Press, 1987; trad. italiana con introd. di G. Galasso. Napoli: Guida, 1995.

Bersani, Mauro. "Alla ricerca dello specifico testuale nelle farse del Sannazaro." Lettere Italiane 34 (1982): 505-529.

. "Farsa, intermezzo, gliommero. Appunti sul teatro del regno aragonese di Napoli." Studi e problemi di critica testuale 26 (1983): 59-77.

. "Un contributo allo studio della lingua del Sannazaro: le farse." Studi di grammatica italiana 11 (1982): 89-99.

Bianchi, Patricia. "Le farse di Iacopo Sannazaro: sondaggi linguistici e tracce intertestuali." Iacopo Sannazaro. La cultura napoletana nell'Europa del Rinascimento. Atti del convegno internazionale di studi (Napoli, 27-28 marzo 2006), a cura di P. Sabbatino. Firenze: Olschki, 2009. 59-69.

Bolzoni, Lina. "Oratoria e prediche." Letteratira italiana II. Torino: Einaudi, 1984. 1041-1074.

Bortoletti, Francesca. L'attore del Parnaso. Ritratti di attori-musici e drammaturgie d'occasione, Milano: Mimesis, 2012.

Egloga e spettacolo nel primo Rinascimento. Da Firenze alle corti. Roma: Bulzoni, 2008.

"Serafino Aquilano and the Mask of Poeta: A Denunciation in the Eclogue of Tyrinto e Menandro (1490)." Voices and Texts in Early Modern Italian Society, a cura di S. Dall'Aglio, B. Richardson, M. Rospocher (Aldershot: Ashgate, 2016 in corso di stampa).

. "Per una nuova drammaturgia. L'egloga nel Quattrocento italiano."

Quaderni d'Italianistica, XXX, 1 (2009): 67-108.

. "Arcadia, festa e performance alla corte dei Re d'Aragona." The Italianist, 36.1 (2016) in corso di stampa.

Bryce, Judith. "Between two friends? Two letters of Ippolita to Lorenzo de' Medici." Renaissance Studies XXI, 3 (2007): 340-365.

Calmeta, Vincenzo. Prose e lettere edite e inedite (con due appendici di altri inediti), a cura di C. Grayson. Bologna: Commissione per i testi di lingua, 1959.

Caracciolo Aricò, Angela. "Il mito di Orfeo nel mondo aragonese." Critica letteraria 137, 4 (2007): 627-49.

Cattin, Giulio. "II Quattrocento.” Letteratura Italiana, a cura di A. Asor Rosa, vol. VI, Teatro, musica, tradizione dei classici. Torino: Einaudi, 1986. 273-313. 
Cicali, Gianni. "Strategie drammaturgiche di un contemporaneo di Goldoni. Pietro Trinchera (1702-1755)." Problemi di critica goldoniana 8 (2001): 133-201.

Ciminelli, Serafino (Aquilano). Sonetti e altre rime, a cura di A. Rossi. Roma: Bulzoni, 2005. . Strambotti, a cura di A. Rossi. Milano: Guanda, 2002.

Colangelo, Francesco. Vita di Antonio Beccadelli, soprannominato il Panormita, Napoli: Angelo Trani, 1820.

Corti, Maria. "Il codice bucolico e l'Arcadia di Jacobo Sannazaro." Metodi e fantasmi. Milano: Feltrinelli, 2001. 283-304

Crispo, Battista. Vita di Giacopo Sannazaro, descritta da Gio. Battista Crispo, da Gallipoli. Di nuovo ristampata, et accresciuta. Roma: presso à Luigi Zannetti, 1593.

Croce, Benedetto. I teatri di Napoli dal Rinascimento alla fine del secolo decimottavo. Napoli: Pierro, 1891.

. "Un gliommero inedito del Quattrocento." Archivio storico per le province napoletane. N.s. 41 (1916): 138-145.

Cronica di Napoli del notar Giacomo, a cura di P. Garzilli. Napoli: Stamperia Reale, 1845.

Cruciani, Fabrizio. Il teatro nel Rinascimento, Roma 1450-1550. Roma: Bulzoni, 1983.

"Il teatro e la festa." Il teatro italiano del Rinascimento, a cura di F. Cruciani e D. Seragnoli. Roma: Bulzoni, 1987. 31-52.

D’Alos-Moner, Ramòn. "Documenti per la storia della Biblioteca di Alfonso il Magnanimo." Miscellanea Francesco Erle, 5 voll. Roma: 1924. Vol. 5, 390-422.

De Blasi, Nicola (a cura di). Lo gliommero napoletano Licinio se 'l mio inzegno. Napoli: Libreria Dante \& Descartes, 1999.

"A proposito degli gliommeri dialettali di Sannazaro: ipotesi di una nuova attribuzione." Iacopo Sannazaro. La cultura napoletana, a cura di Pasquale Sabbatino. Firenze: Olschki, 2009. 29-57.

. "Intrattenimento letterario e generi conviviali (farsa, intramesa, gliommero) nella Napoli aragonese." Passare il tempo. La letteratura del gioco e dellintrattenimento, Atti del convegno internazionale di Pienza. Roma: Salerno, 1993. 129-159.

De Blasis, Giuseppe. Racconti di storia napoletana. Napoli: Perrella, 1908. 
De Filippis, Domenico e Nuovo, Isabella. "Tra cronaca e storia: le forme della memoria nel Mezzogiorno.” La Memoria e la Città, a cura di C. Bastia e M. Bolognini. Bologna: 1995. 419-66.

De Maio, Giuniano. De Maiestate, a cura di Dante Gaeta. Bologna: Commissione per i testi in lingua, 1956.

De Marinis, Tammaro e Perosa, Alessandro (a cura di). Nuovi documenti per la storia del Rinascimento (sezione Aragonesia). Firenze: Olschki, 1970.

De Marinis, Tammaro. La biblioteca napoletana dei re d'Aragona. 4 voll. Supplemento, II. Verona: 1969.

Delle Donne, Roberto. "La corte napoletana di Alfonso il Magnanimo: il mecenatismo regio." La Corona de Aragón en el centro de su Historia 1208-1458. La Monarchia Aragonesa y los Reinos dela Corona. Zaragoza: Gobierno de Aragón, 2010, distribuito in formato digitale da Reti Medievali, www.retimedievali.it. 255-270.

Deramaix, Marc. “'Excellentia et Admiratio' dans l'Actius de Giovanni Pontano." Mélanges de l'Ecole française de Rome. Moyen-Age, Temps modernes 99 (1987): 171-212.

Dionisotti, Carlo. "Appunti sulle rime del Sannazaro." Giornale Storico della Letteratura Italiana CXL (1963): 161-211.

Falletti, Clelia. "Le feste per Eleonora d'Aragona da Napoli a Ferrara (1473)." Guarino 1988, 121-140, e ripreso in parte in Bortoletti 2012: 199-208.

Facio, Bartolomeo. Rerum gestarum Alfonsi regis libri. Testo latino, trad. italiana, commento e introd. a cura di D. Pietragalla. Alessandria: Edizioni dell'Orso, 2004.

Fenzi, Enrico. "Cariteo: il fascino del nome.” Il nome del testo VII (2005): 49-76. . “'Et havrà Barcellona il suo poeta.' Benet Garret, il Cariteo." Quaderns d'Italia 7 (2002): 117-140.

Ferraù, Giacomo. Il tessitore di Antequera. Storiografia umanistica meridionale. Roma: Istituto storico italiano per il Medioevo, 2001.

Flamini, Francesco. La lirica Toscana del Rinascimento anteriore ai tempi del Magnifico. Pisa: 1981, rist. anast. Firenze: Le lettere 1977.

"Francesco Galeota gentiluomo napoletano del Quattrocento e il suo inedito canzoniere." Giornale storico della letteratura italiana XX (1892): $1-90$.

Fuscano, Ian Bernardino. Stanze sovra la bellezza di Napoli, a cura di C. A. Addesso. Napoli: Edizioni Scientifiche Italiane, 2007. 
Galasso, Giuseppe. "Da 'Napoli gentile' a 'Napoli fedelissima,"” in Id. Napoli capital. Identità politica e identità cittadina. Studi e ricerche 1266-1860, Napoli: Electa, 1998: 61-110.

Gareth, Benedetto (Cariteo). Le Rime di Benedetto Gareth detto il Caritheo. Secondo le due stampe originali. Introduzione e note a cura di E. Pèrcopo. Napoli: 1892.

Gerbino, Giuseppe. Music and the Myth of Arcadia in Renaissance Italy. Cambridge: University of Cambridge, 2009. . "Il canto di Serafino e il dilemma degli umanisti." L'attore del Parnaso. Ritratti di attori-musici e drammaturgie d'occasione a cura di F. Bortoletti, Milano: Mimesis, 2012: 315-343.

Giustiniani, Vito Rocco. "Serafino Aquilano e il teatro del Quattrocento." Rinascimento II, 4 (1965): 101-117.

Guarino, Raimondo (a cura di). Teatro e culture della rappresentazione. Lo spettacolo in Italia nel Quattrocento. Bologna: il Mulino, 1988.

. Teatro e mutamenti. Rinascimento e spettacolo a Venezia. Bologna: il Mulino, 1995.

"Figures et mythes de la musiques dans les spectacles de la Renaissance italienne." Imago Musicae XVI-XVII (1999-2000): 11-24.

"Archeologia e spettacolo a Roma nell'età di Giulio II." Metafore di un pontificato. Giulio II (1503-1513). Atti del Convegno, Roma 2-4 dicembre 2008, a cura di F. Cantatore et al. Roma: Roma nel Rinascimento, 2010. $345-364$

Haraszti, Emilie. "Les musiciens de Mathias Corvin et de Beatrice d'Aragon." La Musique instrumentale de la Renaissance, a cura di J. Jacquot. Paris: Editions Du Centre National de la Recherche Scientifique, 1955. 34-59.

Iacono, Antonietta. "Il trionfo di Alfonso d'Aragona tra memoria classica e propaganda di corte." Rassegna storica salernitana 51 (2009): 9-57.

Ianziti, Gary. Humanistic historiography under the Sforzas. Politics and propaganda in 15th century Milan. Oxford: Clarendon Press; New York: Oxford University Press, 1988.

Leostello, Jeompiero. "Effemeridi delle cose fatte per il duca di Calabria (14841491) di Joampiero Leostello." Documenti per la storia le arti e le industrie delle provincie napoletane, a cura di Gaetano Filangieri. Napoli: Tip. Dell'Accademia Reale delle Scienze, 1883. 
Lettere inedite di Joviano Pontano in nome dé reali di Napoli, a cura di F. Gabotto, "Scelta di curiosità letterarie inedite o rare dal secolo XIII al XIX, Dispensa CCXLV.” Bologna: Romagnoli Dall'Acqua, 1893.

Mayer, Elizabeth. "Un umanista fiorentino alla corte di Mattia Corvino." Studi e documenti italo-ungheresi dell'Accademia d'Ungheria di Roma II (1938): $123-167$.

Napoli-Signorelli, Pietro. Vicende della coltura delle Due Sicilie, voll. 4. Napoli: Flauto, 1785.

Natale, Michele. Antonio Beccadelli detto il Panormita. Caltanisettta: Tipogr. dell'Omnibus, 1902.

Nevola, Maria Luisa. "Le farse di Pietro Antonio Caracciolo." In Ead. Il silenzio in Manzoni e altri saggi, a cura di M. Montanile. Cava de' Tirreni: Avagliano, 1995. 59-68.

Nocilli, Cecilia. "La Presa di Granata e il Triunfo de la Fama. Danza, musica e politica nelle farse di Iacopo Sannazaro (1492)." Il mito d'Arcadia. Pastori e Amori nelle arti del Rinascimento, Atti del convegno, a cura di D. Boillet e A. Pontremoli. Firenze: Olschki, 2007. 167-188.

Notturno, Napolitano. Comedia noua de Notturno Napolitano intitolata Gaudio d'amore. Venezia: C. Stampone, 1526.

- Triumphi de gli mirando spettaculi et ricche vivande dil solenne convivio fatto da sacri romani al magnifico Iuliano et invicto Laurentio de Medici con il resto creato il sommo pontefice Leon Decimo, con tutta la genealogia et Gloria de Firenza e Roma: composti per Nocturno Neapolitano. Ioannis Thomae Montecatini Adernionis Comitis De Vita Illustris Constantiae Davalos Comitissae Acerrarum, Roma: A Blado, 1539.

Ong, Walter. Oralità e scrittura. Le tecnologie della parola. Bologna: il Mulino, 1986.

Padoan, Giorgio. La commedia rinascimentale veneta. Vicenza: Neri Pozza, 1982.

Panormita, Il (Beccadelli, Antonio detto il). De dictis et factis Alphonsi regis Aragomum (1455), Basel: 1538; testo latino dell'opera consultabile in internet: http://www.bibliotecaitaliana.it, dell'Università degli studi di Roma.

Parenti, Giovanni. "Antonio Caracciolo.” Dizionario Biografico degli Italiani, vol. 19, 1976.

. "Un gliommero di P. J. De Jennaro: Eo non agio figli ne fittigli." Studi di filologia italiana 36 (1978): 321-365. 
“'Antonio Carazolo desamato.' Aspetti della poesia volgare aragonese nel ms. Riccardiano 2752.” Studi di filologia italiana 37 (1979): 119-279. . Benet Garret detto il Cariteo. Profilo di un poeta. Firenze: Olshki, 1993.

Pellegrino, Gaspare. Historia Alphonsi primi regis, a cura di F. Delle Donne. Firenze: SISMEL edizioni del Galluzzo, 2007.

Pèrcopo, Erasmo. "Marc'Antonio Epicuro." Giornale Storico della Letteratura Italiana XII (1888): 1-76.

Pieri, Marzia. La scena boschereccia nel Rinascimento italiano. Padova: Liviana, 1983.

"“Sumptuosissime pompe': lo spettacolo nella Napoli aragonese." Studi di filologia offerti dagli allievi a Lanfranco Caretti. vol. 1. Roma: Salerno, 1985. 39-85.

. "Dalla lirica alla festa: il caso dell'egloga nella lirica aragonese." Origini del dramma pastorale in Europa. Atti del convegno internazionale a cura di M. Chiabò e F. Doglio. Viterbo: Union Printing, 1985. 71-89.

Perleoni, Giuliano (detto il Rustico Romano). "Canzone V de nuova textura al Illustrissimo S. Duca di Calabria recitata in un convito in forma d'un pastore in la recuperatione d'Hydronto." R. Romano, Compendio di sonetti et alter rime de varie texture intitolato il Perleone. Napoli: Aiolfo de Cantono, 1492.

Pontano, Giovanni. Dialoghi, a cura di C. Previtera. Firenze: Sansoni, 1943.

. Trattati delle virtù speciali, a cura di F. Tateo. Roma: Bulzoni, 1999. . De Principe, a cura di G.M. Cappelli. Roma: 2003.

- Aegidius. Dialogo, a cura di F. Tateo. Roma: Arbor Sapoentiae, 2014.

Pontieri, Erenesto. Alfonso il Magnanimo re di Napoli (1435-1458). Napoli: Edizioni Scientifiche Italiane, 1975.

Raina, Pio. Il padiglione di Re Alfonso. Firenze: Tipografia Galileiana, 1904.

Richardson, Brian. "Sixteenth-Century Italian Petrarchists and Musical Settings of their Verse." Voices and Texts in Early Modern Italian Society, a cura di S. Dall'Aglio, B. Richardson, M. Rospocher (Aldershot: Ashgate, 2016 in corso di stampa).

Rogeri De Piacenza di Nardò. Opere (cod. per F 27), a cura di M. Marti. Lecce: Milella, 1977.

Riccò, Laura. Dalla zampogna all'aurea cetra. Egloghe, pastorali, favole in musica. Roma: Bulzoni, 2015.

Rossi, Antonio. Serafino Aquilano e la poesia cortigiana. Brescia: Morcelliana, 1980. 
Ruffini, Franco. Commedia e festa nel Rinascimento. La "Calandria" alla corte di Urbino. Bologna: il Mulino, 1986.

Ryder, Alan. The kingdom of Naples under Alfonso the Magnanimous. Oxford: Clarendon Press, 1976.

. "Antonio Beccadelli: A Humanist in Goverment." Cultural Aspect of Italian Renaissance: Essays in Honour of Paul Oskar Kristeller, a cura di C.H. Clough. Manchester: 1976. 123-140.

Sabadino degli Arienti, Giovanni. "De Hyppolita Sphorza, duchessa di Calabria." Gynevra de le clare donne, a cura di C. Ricci e A. Bacchi della Lega, Scelta di curiosità letterarie inedite o rare dal secolo XIII al XVII, Dispensa CCXXIII. Bologna: Romagnoli Dall'Acqua, 1888. 336-352.

Sannazaro, Iacopo. Opere volgari, a cura di A. Mauro. Bari: Laterza, 1961.

Santagata, Marco. La lirica aragonese: studi sulla poesia napoletana del secondo Quattrocento. Padova: Antenore, 1979.

Santoro, Marco. "Il Panormita 'aragonese." Esperienze letterarie 9 (1984): 3-24.

Soranzo, Matteo. "Reading Marsilio Ficino in Renaissance Italy. The case of Aragonese Naples." Quaderni d'Italianistica, 33.1 (2012): 27-46.

. "Audience and Quattrocento Pastoral: the Case of Jacopo Sannazaro's Arcadia." Skepsi, num. monog. Consideration of Audience in Early and Modern Medieval Studies, 2. 1 (2009): 49-65.

Summonte, Giovanni Antonio. Dell'Historia della città, e Regno di Napioli, 4 voll. Napoli: A spese di Antonio Bulifon libraro all'insegna della sirena, l'anno santo, 1675.

Tateo, Francesco. Umanesimo etico di Giovanni Pontano. Lecce: Milella, 1972.

Torraca, Francesco. "Pier Antonio Caracciolo." Studi di storia letteraria napoletana. Livorno: Vigo, 1884: 65-81.

- "Li gliommeri di Iacopo Sannazaro." Giornale storico della letteratura italiana 4 (1884): 209-228; poi con il titolo di "I gliommeri di Iacopo Sannazaro." Aneddoti di storia letteraria napoletana. Città di Castello: Il solco, 1925. 321-365.

Ventrone, Paola. "Feste e spettacoli nella Firenze di Lorenzo il Magnifico." Ead., Le temps revient 1992 . 38-48.

. Gli araldi della commedia. Teatro a Firenze nel Rinascimento. Pisa: Pacini, 1993.

. Le temps revient - Il tempo si rinnova. Feste e spettacoli nella Firenze di Lorenzo. Milano: Silvana edit., 1992. 
- "Civic Performance' in Renaissance Florence." Voices and Texts in Early Modern Italian Society, a cura di S. Dall'Aglio, B. Richardson, M. Rospocher (Aldershot: Ashgate, 2016 in corso di stampa).

Veronese, Guarino. Epistolario, a cura di R. Sabbadini, 3 voll. Venezia: 1915-1920.

Villani, Gianni. "Sul 'manoscritto base' del Libro pastorale nominato Arcadio." Per Leggere, XII 23 (2012): 111-152. . Per l'edizione dell'Arcadia. Roma: Salerno, 1989.

Vita di Antonio Beccadelli soprannominato il Panormita, a cura di Francesco Colangelo. Napoli: Trani, 1820.

Welch, Evelyn S. "Between Milan and Naples: Ippolita Maria Sforza, Duchess of Calabria." The French Descent into Renaissance Italy 1494-1495, a cura di David Abulafia. Aldershot: Variorum, 1995. 123-136.

Zorzi, Ludovico. Il teatro e la città. Saggi sulla scena italiana. Torino: Einaudi, 1977.

Zumthor, Paul. La presenza della voce. Introduzione alla poesia orale. Bologna: il Mulino, 1984. 